\title{
Structure-Preserving Discretization of Incompressible Fluids
}

\author{
Thesis by \\ Dmitry Pavlov \\ In Partial Fulfillment of the Requirements \\ for the Degree of \\ Doctor of Philosophy
}

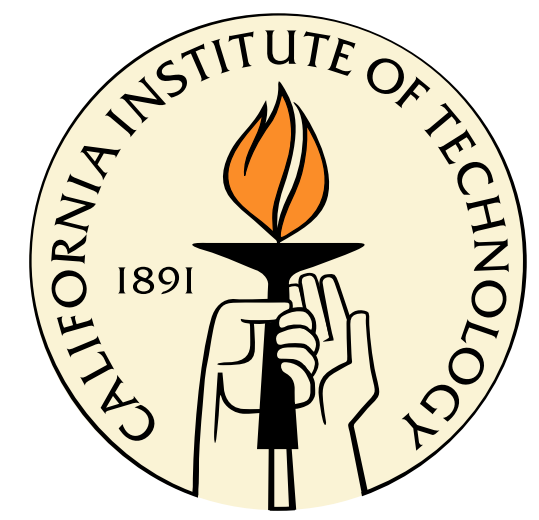

California Institute of Technology

Pasadena, California

2009

(Defended May 13, 2009) 
(c) 2009

Dmitry Pavlov

All Rights Reserved 


\section{Acknowledgments}

I am deeply grateful to my advisors, Jerrold Marsden and Mathieu Desbrun for their guidance, support and encouragement; and for giving me freedom to be creative in my research. It has been a true honor to work with them.

I would like to thank my collaborators, Patrick Mullen, Yiying Tong, Eva Kanso and Keenan Crane for their incredible help in developing this work. I'm also grateful to Yann Brenier, Tudor Ratiu and Nikolai Makarov for inspiring conversations and insightful remarks.

I wish to express my gratitude to all members of the Caltech community, in particular in the departments of Mathematics, CDS and CS, for making Caltech an ideal place to work.

I would like to thank my family and friends for their support and encouragement. Most of all, I want to thank my girlfriend Anna for her love and patience. 


\section{Abstract}

The geometric nature of Euler fluids has been clearly identified and extensively studied in mathematics. However computational approaches to fluid mechanics, mostly derived from a numerical-analytic point of view, are rarely designed with structure

preservation in mind, and often suffer from spurious numerical artifacts. In contrast, we geometrically derive discrete equations of motion for fluid dynamics from first principles. Our approach uses a finite-dimensional Lie group to discretize the group of volume-preserving diffeomorphisms, and the discrete Euler equations are derived from a variational principle with non-holonomic constraints. The resulting discrete equations of motion induce a structure-preserving time integrator with good longterm energy behavior, for which an exact discrete Kelvin circulation theorem holds. Possible extensions of our method to magnetohydrodynamics, viscous flows, optimal transport and a link to Brenier's generalized flows are also discussed. 


\section{Contents}

Acknowledgments $\quad$ iv

Abstract $\quad$ v

1 Introduction 1

1.1 Euler Equations for Ideal Incompressible Fluid . . . . . . . . . . . . . 2

1.1.1 Configuration space of incompressible fluids . . . . . . . . . 2

1.1.2 Velocity field and metric on SDiff . . . . . . . . . . . . 3

1.1.3 Variational principle and the Euler equation . . . . . . . . . . 4

1.2 Overview of Results . . . . . . . . . . . . . . . . 6

1.2.1 Notations ..................... . . 9

2 Discrete Incompressible Fluids $\quad 11$

2.1 Discrete Volume-Preserving Diffeomorphisms . . . . . . . . . . . . . . 11

2.1.1 Finite-dimensional configuration space . . . . . . . . . . . 11

2.1.2 Discrete velocity field . . . . . . . . . . . . . . . . 15

2.1.3 Discrete commutator . . . . . . . . . . . . . . . . 18

2.1.4 Non-holonomic constraints . . . . . . . . . . . . . . . . . . . . 19

2.1.5 Relation between $A$ and Fluxes . . . . . . . . . . . . . . . . 20

2.1.6 Lagrangian dynamics with non-holonomic constraints on $\mathcal{D}(\mathcal{M}) \quad 21$

2.2 Structure-Preserving Spatial Field Discretization . . . . . . . . . . . . 24

2.2.1 Discrete zero-forms . . . . . . . . . . . . . . 24

2.2.2 Discrete one-forms . . . . . . . . . . . . . . . . 25

2.2.3 Discrete two-forms ................. 26 
vii

2.2.4 Other operators on discrete forms . . . . . . . . . . . . 27

2.2.5 Discrete flat operator . . . . . . . . . . . . 29

2.3 Dynamics on the Group of $\Omega$-Orthogonal Stochastic Matrices . . . . . 40

2.3.1 Variational principle ................ . 40

2.3.2 Discrete Kelvin's theorem . . . . . . . . . . . . . . . . . . 45

2.3.2.1 Continuous property ............. 45

2.3.2.2 Discrete Kelvin's theorem . . . . . . . . . . . . 46

2.4 Fluid Evolution in Discrete Time . . . . . . . . . . . . . . . . 50

$\begin{array}{lll}3 & \text { Conclusion } & 58\end{array}$

3.1 Shortest Paths and Generalized Flows . . . . . . . . . . . . . 58

3.1.1 Shnirelman's example. . . . . . . . . . . . . . 59

3.1.2 Generalized flows . . . . . . . . . . . . . . . . . 60

3.1.3 Discrete generalized flows . . . . . . . . . . . . 61

3.2 Symplecticity . . . . . . . . . . . . . . . . . . . 62

3.3 Optimal Transport ................... 63

3.4 Other Future Work . . . . . . . . . . . . . . . . . 63

3.4.1 Magnetohydrodynamics ............. 63

3.4.2 Viscosity and subscale modeling . . . . . . . . . . . . 63

$\begin{array}{lr}\text { Bibliography } & 64\end{array}$ 


\section{Chapter 1}

\section{Introduction}

While the geometric nature of Euler fluids has been extensively studied in mathematics, its differential standpoint sharply contrasts with the numerical approaches traditionally used in Computational Fluid Dynamics (CFD). In particular, methods based on particles, vortex particles, staggered Eulerian grids, spectral elements, as well as hybrid Lagrangian-Eulerian formulations were not designed with structure preservation in mind - in fact, recent work (e.g., from Tom Hou [22]) pinpoints the loss of Lagrangian structures as a major numerical impediment of current CFD techniques. In contrast, structure preserving methods (so-called geometric integrators) have recently been popular in the context of Lagrangian dynamics in solid mechanics. Based on discrete versions of Hamilton's principle and its variants, they have been shown to capture the dynamics of the mechanical system they discretize without the traditional numerical artifacts such as loss of energy or momenta.

While the variational principles for incompressible fluid mechanics are best expressed in a Lagrangian formalism, computational efficiency calls for an Eulerian treatment of fluid computations to avoid numerical issues inherent to deforming meshes. In order to circumvent these issues, a new Eulerian formulation of discrete fluid mechanics is thus needed.

Guided by the variational integrators used in the Lagrangian setting, this dissertation introduces a discrete, structure-preserving theory for incompressible perfect fluids based on Hamilton-d'Alembert's principle. Such a discrete variational approach to fluid dynamics guarantees invariance under the particle-relabeling group action, thus 
giving rise to a discrete form of Kelvin's circulation theorem. Also due to their variational character, the resulting numerical schemes exhibit good long-term energy and circulation behavior.

\subsection{Euler Equations for Ideal Incompressible Fluid}

In this section we describe the continuous derivation of Euler equation from a geometric, variational principle first formulated by Newcomb [32] (see also [10]) and generalized to the case of general Lie algebras by Marsden and colleagues [11, 27]. Our discretization will basically follow the same derivation, but in a discrete setting.

\subsubsection{Configuration space of incompressible fluids}

Consider a fluid occupying a domain $M \in \mathbb{R}^{n}$. To describe the motion of this fluid we have to determine the position of every particle in time. A map $g_{t}: M \rightarrow M$ can be used to indicate that a fluid particle located at point $x$ at time $t=0$ has moved to point $g_{T}(x)$ at time $t=T$. Naturally, we will restrict these maps to be smooth with their inverse, i.e., to be diffeomorphisms. Since we only consider incompressible fluids, the diffeomorphisms $g_{t}$ we will be using have to preserve volume on $M$ :

$$
\operatorname{vol}\left(g_{t}^{-1}(U)\right)=\operatorname{vol}(U), \quad \text { for every } U \subset M,
$$

where $\operatorname{vol}($.$) is the Lebesgue measure on M \subset \mathbb{R}^{n}$. The motion of an incompressible fluid is thus described by a curve $g_{t}$ on the space of volume-preserving diffeomorphisms (see [1], [2] and [5]). Notice that not every volume-preserving diffeomorphism represents a valid configuration of an incompressible fluid: only diffeomorphisms that can be connected to the identity map by a continuous path are relevant; for example, a diffeomorphism reversing orientation cannot be reached. So the configuration space of incompressible fluids is the connected component containing the identity of the space of volume-preserving diffeomorphisms, which will be denoted by $\operatorname{SDiff}(M)$. 


\subsubsection{Velocity field and metric on SDiff}

Consider a flow $\left\{g_{t}\right\}_{t \in[0, T]} \subset \operatorname{SDiff}(M)$. At any point $x \in M$ we can define the velocity $v(t, x)$ of a particle passing through $x$ at time $t$ :

$$
v(t, x)=\dot{g}_{t}\left(g_{t}^{-1}(x)\right)
$$

We will call the (time-dependent) vector field $v(t, x)$ the Eulerian velocity field of our incompressible fluid. And since $g_{t}$ is volume preserving, the vector field $v(t, x)$ has to be divergence-free. The set of all divergence-free vector fields will be denoted by $\operatorname{SVect}(M)$. Now, as the space $\operatorname{SVect}(M)$ has its $L_{2}$-norm

$$
\|v\|^{2}=\int_{M} v^{2}(x) d x
$$

we can define the action and the length of a path as follows.

Definition 1. The action along a path $\left\{g_{t}\right\}_{t \in[0, T]} \subset \operatorname{SDiff}(M)$ is

$$
\mathcal{L}\left\{g_{t}\right\}_{0}^{T}=\frac{1}{2} \int_{0}^{T}\left\|\dot{g}_{t}\right\|_{L_{2}}^{2} d t
$$

Definition 2. The length of a path $\left\{g_{t}\right\}_{t \in[0, T]} \subset \operatorname{SDiff}(M)$ is the average distance traveled by the particles:

$$
\ell\left\{g_{t}\right\}_{0}^{T}=\frac{1}{2} \int_{0}^{T}\left\|\dot{g}_{t}\right\|_{L_{2}} d t
$$

It is easy to see that length and action of a path are related to each other by the following inequality:

$$
\ell^{2} \leq 2 \mathcal{L} T
$$

and that the action of a path depends on the parametrization while length does not.

Now we can define a metric on $\operatorname{SDiff}(M)$ as the infimum of lengths of paths connecting two given diffeomorphisms $g, h \in \operatorname{SDiff}(M)$ :

$$
\operatorname{dist}_{\mathrm{SDiff}}(g, h)=\inf _{\substack{\left\{g_{t}\right\} \subset \operatorname{SDiff}(M) \\ g_{0}=g, g_{1}=h}} \ell\left\{g_{t}\right\}_{0}^{1} .
$$




\subsubsection{Variational principle and the Euler equation}

A path $\left\{g_{t}\right\}$ with $g_{0}=$ Id describes the motion of an ideal incompressible fluid if it is a critical point of the action functional $\mathcal{L}$. We will derive the Euler equations for ideal incompressible fluids from this variational principle.

Let's consider a diffeomorphism $h \in \operatorname{SDiff}(M)$ and a family of paths $\left\{g_{t}^{s}\right\} \subset$ $\operatorname{SDiff}(M)$ such that $g_{0}^{s}=\operatorname{Id}$ and $g_{T}^{s}=h$. We use $\delta$ to denote variation, i.e., derivative with respect to the parameter $s: \delta g_{t}^{s}=\frac{\partial g_{t}^{s}}{\partial s}$. Our goal is to find a path $\left\{g_{t}\right\} \subset \operatorname{SDiff}(M)$ satisfying

$$
\delta \mathcal{L}\left\{g_{t}\right\}_{0}^{T}=0, \quad g_{0}=\mathrm{Id}, g_{T}=h
$$

To solve this equation, notice that since any $g_{t}$ is volume-preserving the action can be rewritten in terms of the Eulerian velocity:

$$
\mathcal{L}\left\{g_{t}\right\}_{0}^{T}=\frac{1}{2} \int_{0}^{T} \int_{M} v(t, x)^{2} d x d t
$$

where $v(t, x)=\dot{g}_{t}\left(g_{t}^{-1}(x)\right)$. Thus, Equ. (1.5) can be written as

$$
\delta \frac{1}{2} \int_{0}^{T} \int_{M} v(t, x)^{2} d x d t=\int_{0}^{T} \int_{M}(v(t, x), \delta v(t, x)) d x d t=0
$$

The following lemma gives the specific form that the variation $\delta v$ must have.

Lemma 1. Variation $\delta v$ satisfies the Lin constraints:

$$
\delta v=\dot{\xi}+[v, \xi]
$$

where $\xi \in \operatorname{SVect}(M), \xi(0, x)=\xi(T, x)=0$ and $[\cdot, \cdot]$ is the commutator co $^{1}$ vector fields.

This lemma can be proved by explicitly writing the derivative:

$$
\delta v(t, x)=\frac{\partial}{\partial s} \dot{g}_{t}^{s}\left(\left(g_{t}^{s}\right)^{-1}(x)\right) .
$$

\footnotetext{
${ }^{1}$ See [25] for the definition and properties of this commutator.
} 
A proof in case of a general Lie group can be found in [27].

Now, we can use the Lin constraints to find a solution to the variational problem expressed in Eq. (1.7). Recalling that the inner product of vector fields can be written as the pairing of a vector field and a 1-form

$$
(u, v)=\left\langle u^{b}, v\right\rangle=\left\langle u, v^{b}\right\rangle
$$

we have:

$$
\begin{aligned}
\delta\left(\frac{1}{2} \int_{0}^{T} \int_{M} v^{2} d x d t\right)= & \int_{0}^{T} \int_{M}\left\langle v^{b}, \delta v\right\rangle d x d t= \\
& \int_{0}^{T} \int_{M}\left\langle v^{b}, \dot{\xi}\right\rangle d x d t+\int_{0}^{T} \int_{M}\left\langle v^{b},[v, \xi]\right\rangle d x d t .
\end{aligned}
$$

Since $[v, \xi]=L_{v} \xi$, we can use the properties of the Lie derivative to write

$$
\left\langle v^{b},[v, \xi]\right\rangle=\left\langle v^{b}, L_{v} \xi\right\rangle=-\left\langle L_{v} v^{b}, \xi\right\rangle
$$

Using this equality and integration by parts, we get

$$
\delta\left(\frac{1}{2} \int_{0}^{T} \int_{M} v^{2} d x d t\right)=-\int_{0}^{T} \int_{M}\left\langle\dot{v}^{b}, \xi\right\rangle d x d t-\int_{0}^{T} \int_{M}\left\langle L_{v} v^{b}, \xi\right\rangle d x d t
$$

Thus, in order to solve the variational problem, we need to find $v \in \operatorname{SVect}(M)$, which satisfies

$$
\int_{0}^{T} \int_{M}\left\langle\dot{v}^{b}+L_{v} v^{b}, \xi\right\rangle d x d t=0
$$

for every $\xi(t, x) \in \operatorname{SVect}(M), \xi(0, x)=\xi(T, x)=0$, which is equivalent to

$$
\int_{M}\left\langle\dot{v}^{b}+L_{v} v^{b}, \xi\right\rangle d x=0, \quad \text { for every } \xi \in \operatorname{SVect}(M)
$$

This implies that the form $\dot{v}^{b}+L_{v} v^{b}$ is a gradient of some function:

$$
\dot{v}^{b}+L_{v} v^{b}=-d \bar{p}
$$


The reader will recognize the Euler equation in terms of the one-form $v^{b}$. We can further use the identity:

$$
L_{v} v^{b}=\left(\nabla_{v} v\right)^{b}+\frac{1}{2} \mathbf{d}(v, v)
$$

to rewrite the equation of motion as

$$
\dot{v}+\nabla_{v} v+\nabla p=0
$$

Note that $p$ corresponds to the conventional pressure, while $\bar{p}$ is the modified pressure, which differs from the pressure by the value of kinetic energy:

$$
p=\bar{p}+\frac{1}{2}(v, v)
$$

\subsection{Overview of Results}

The goal of this thesis is to formulate a discrete analog of ideal incompressible fluids that preserves underlying group structures. To achieve this goal we will discretize the domain of motion $M$ with a mesh $\mathbb{M}$ to introduce a finite-dimensional discretization of the configuration space $\operatorname{SDiff}(M)$, along with a Lagrangian defined on it. Applying Hamilton-d'Alembert's principle in this discrete setting will lead to finite-dimensional dynamics approximating the dynamics of the Euler equations. While our exposition will make no restriction on the mesh, our approach can easily be explained for regular grids in the following fashion:

- The first step of our work is to discretize $\operatorname{SDiff}(M)$ into a finite-dimensional Lie group, along with a discrete action, in such a way that the corresponding discrete Euler-Lagrange equations converge to the continuous Euler equations. To construct our finite-dimensional approximation of SDiff, we will first note that for any $g \in$ SDiff there is an operator $U_{g}: \varphi \mapsto \varphi\left(g^{-1}\right)$ that is unitary and preserves constants. We will in fact offer a discrete version of this operator that has these two properties as well, i.e., we will discretize the space 
of volume-preserving diffeomorphisms through the group of orthogonal matrices that preserve constant vectors (i.e., $\left.(1, \cdots, 1)^{t}\right)$. These matrices are signed doubly-stochastic, i.e., their rows and columns all sum to 1 . We will denote by $\mathcal{D}(\mathbb{M})$ the space of orthogonal, signed doubly-stochastic (OSDS) matrices, which will serve as our discrete version of SDiff thereafter.

- By analogy to the continuous case, the motion of a discrete fluid will be described by a curve $q_{t}$ in the space of OSDS matrices. Its Eulerian velocity will be defined as the matrix $A=\dot{q} q^{-1}$. This matrix belongs to the Lie algebra of the OSDS matrices, which consists of antisymmetric "doubly-null" matrices, i.e., matrices whose rows and columns sum to 0 . Such a matrix $A$ will be thus considered a discrete version of the continuous vector field $v$. Since the matrix elements $A_{i j}$ describe infinitesimal exchanges of mass between cells $C_{i}$ and $C_{j}$, we will show that it is natural (and computationally more efficient) to restrict ourselves to matrices, which have nonzero elements only for neighboring cells $C_{i}$ and $C_{j}$. By doing so, we will introduce nonholonomic constraints (abbreviated as NHC).

- To define a Lagrangian on $T \mathcal{D}(\mathbb{M})$ we will note that the Lagrangian for an ideal fluid can be written as $L=\frac{1}{2} \int \mathbf{i}_{v} v^{\mathrm{b}} d x$, where $v^{\mathrm{b}}$ is the one-form associated with $v$. We can then formally define discrete $k$-forms as simple $(k+1)$-tensors, and we can also derive the operators of contraction $\mathbf{i}$ and differentiation $\mathbf{d}$ acting on these tensors. A discrete Lie derivative will then be obtained using Cartan's formula $L_{v}=\mathbf{i}_{v} \mathbf{d}+\mathbf{d} \mathbf{i}_{v}$ (note that this definition will coincide with the discrete notion of advection that derives from Hamilton-d'Alembert's principle). Finally, with a discrete version of the flat operator b, we will define our discrete Lagrangian to be

$$
L=\frac{1}{2} \mathbf{i}_{A} A^{b}=\frac{1}{4} \operatorname{Tr}\left(A^{T} A^{b}\right) .
$$

- We will finally get our discrete Euler equations from the variational Lagranged'Alembert principle with nonholonomic constraints. The resulting discrete in 


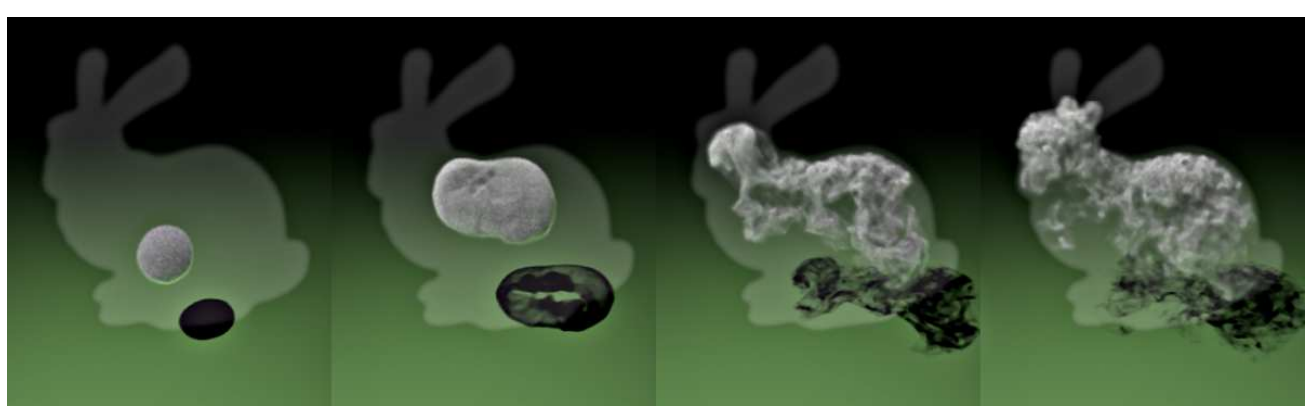

Figure 1.1: A sample simulation: smoke inside a bunny

space and continuous in time version of the Euler equations is

$$
\dot{A}^{b}+L_{A} A^{b}+\mathbf{d} \bar{p}=0
$$

which is strikingly analogous to the continuous equation

$$
\dot{v}^{b}+L_{v} v^{b}+\mathbf{d} \bar{p}=0
$$

A fully discrete variational integrator will be constructed by defining a spacediscrete/time-discrete Lagrangian $L_{d}\left(q_{k}, q_{k+1}\right)=L_{d}\left(A_{k}\right)$. Here, $A_{k}$ is a matrix used to discretely advect $q_{k}$ to $q_{k+1}$ - for example, $A_{k}=\left(q_{k+1}-q_{k}\right) q_{k}^{-1}$ if one uses an explicit Euler integration. The discrete action being

$$
\mathcal{A}_{d}=\sum_{k} L_{d}\left(q_{k}, q_{k+1}\right)
$$

the Euler-Lagrange equations discussed above will become:

$$
\delta \mathcal{A}_{d}=0, \quad \text { where } A_{k} \text { and } \delta q_{k} q_{k}^{-1} \text { satisfy the nonholonomc constraints. }
$$

Solving these equations will provide a variational time update rule $A_{k} \rightarrow A_{k+1}$.

We will also show that applying the derivation of our variational integrator for the simple explicit-Euler definition of $A_{k}$ mentioned above and a regular grid in space leads to an existing energy-preserving finite-difference time integration scheme, 
namely the Harlow-Welsh scheme [17] with Crank-Nicolson time update. However, our approach can be used to derive more general schemes, in particular on arbitrary grids, with similar numerical qualities (see Fig. 1.1 for a result of a simulation).

\subsubsection{Notations}

In this document, we will use $n$ to denote the dimension of the domain (typically, 2 or 3). The letter $\mathbb{M}$ will denote the spatial discretization (mesh), with $N$ being the number of $n$-dimensional cells $\left\{C_{i}\right\}_{i=1 . . N}$ in $\mathbb{M}$. Note that the size of a mesh will refer to the maximum diameter of its cells. We will also use the term regular grid to designate a mesh that consists of cells which are $n$-dimensional cubes of equal size. The notation $N(i)$ will denote the set of indices of cells neighboring cell $C_{i}$, that is, cell $C_{i}$ shares faces only with cells $C_{j}$ with $j \in N(i)$. Finally, Table 1.1 summarizes the various variables used in the remainder of this paper, along with their meaning and representation. 


\begin{tabular}{|c||l|l|}
\hline Symbol & Meaning & Representation \\
\hline \hline$M$ & Domain of motion & $M \subset \mathbb{R}^{n}$ \\
\hline$n$ & Dimension of the domain & $n \in \mathbb{N}$ \\
\hline SDiff $(M)$ & Configuration space for ideal fluid & $\begin{array}{l}\text { Space of volume-preserving dif- } \\
\text { feomorphisms on } M\end{array}$ \\
\hline SVect $(M)$ & $\begin{array}{l}\text { Tangent space of SDiff }(M) \text { at } \\
\text { Identity }\end{array}$ & $\begin{array}{l}\text { Space of divergence-free vector } \\
\text { fields on } M\end{array}$ \\
\hline $\mathbb{M}$ & Mesh & Simplicial or regular mesh \\
\hline$N$ & Number of cells & $N \in \mathbb{N}$ \\
\hline$C_{i}$ & Cell \#i of $\mathbb{M}$ & Tetrahedron or cube in $3 D$ \\
\hline$\Omega$ & Discrete analog of volume form & $\begin{array}{l}\text { Diagonal matrix consisting of vol- } \\
\text { umes of the cells, } \Omega_{i i}=\text { vol }\left(C_{i}\right)\end{array}$ \\
\hline $\mathcal{D}(\mathbb{M})$ & Discrete configuration space & $\begin{array}{l}\text { Space of } \Omega \text {-orthogonal signed sto- } \\
\text { chastic matrices }\end{array}$ \\
\hline $\mathfrak{D}(\mathbb{M})$ & Lie algebra of $\mathcal{D}(\mathbb{M})$ & $\begin{array}{l}\text { Space of } \Omega \text {-antisymmetric null- } \\
\text { column matrices }\end{array}$ \\
\hline$q$ & Discrete configuration & Matrix $\in \mathcal{D}(\mathbb{M}) \subset \mathcal{M}^{N}$ \\
\hline$A$ & Discrete Eulerian velocity $\dot{q} q^{-1}$ & Matrix $\in \mathfrak{D}(\mathbb{M}) \subset \mathcal{M}^{N}$ \\
\hline${ }^{N} F$ & Discrete $k$-form & Tensor of order $(k+1)$ \\
\hline $\mathcal{N}$ & $\begin{array}{l}\text { Space of matrices with sparsity } \\
\text { based on cell adjacency }\end{array}$ & $\begin{array}{l}\text { Constrained set of matrices, } \\
A_{i j} \neq 0 \Rightarrow j \in N(i)\end{array}$ \\
\hline $\mathcal{S}$ & Space of sparse discrete velocities & $\begin{array}{l}\text { Constrained set of velocities, } \mathcal{S}= \\
\mathfrak{D}(\mathbb{M}) \cap \mathcal{N}\end{array}$ \\
\hline
\end{tabular}

Table 1.1: Physical/Geometric meaning of the basic (continuous and discrete) variables used throughout this document. 


\section{Chapter 2}

\section{Discrete Incompressible Fluids}

\subsection{Discrete Volume-Preserving Diffeomorphisms}

We first introduce a finite-dimensional approximation to the infinite-dimensional Lie group of volume-preserving diffeomorphisms that keeps track of the amount of fluid going from one cell to another while preserving two key properties: volume and mass preservation.

\subsubsection{Finite-dimensional configuration space}

Let's now consider that the domain $M$ is approximated by an arbitrary mesh $\mathbb{M}$. Our first step in constructing a discrete representation of ideal fluids is to give an approximation of $\operatorname{SDiff}(M)$ with a finite-dimensional Lie group in such a way that the elements of the corresponding Lie algebra can be considered as discretization of divergence-free vector fields. To achieve this goal, we will discretize not the diffeomorphism $g$ itself, but rather the operator $U_{g}$ associated with it:

$$
\begin{aligned}
U_{g}: & L_{2} \quad \rightarrow L_{2} \\
\varphi(x) & \mapsto \varphi\left(g^{-1}(x)\right) .
\end{aligned}
$$

Here $L_{2}=L_{2}(M, \mathbb{R})$ is the space of real-valued function on $M$ with integrable square. An important property of $U_{g}$ is given by the following lemma. 
Lemma 2 (Koopman's lemma ${ }^{1}$ ). If the diffeomorphism $g$ is volume-preserving, then $U_{g}$ is a unitary operator on $L_{2}$.

Another property of $U_{g}$, important for this work, is constant preservation, i.e., $U_{g} C=C$ for every constant function $C$.

Now let's see how we can discretize the operator $U_{g}$.

Discrete functions. To discretize the operator $U_{g}$ we first need to discretize the space on which $U_{g}$ acts. Since the mesh $\mathbb{M}_{h}$ splits the domain of motion $M$ into $N$ cells $C_{i}$ of maximum diameter $h$, a function $\varphi \in C^{0}(M ; \mathbb{R})$ can be approximated by a step function $\tilde{\varphi}_{h}$, constant within each cell of the mesh, through a projection $R_{\mathbb{M}_{h}}$ :

$$
R_{\mathbb{M}_{h}}: \varphi \mapsto \tilde{\varphi}_{h}, \quad \tilde{\varphi}_{h}=\sum_{i}\left[\frac{1}{\Omega_{i}} \int_{C_{i}} \varphi\right] \chi_{C_{i}},
$$

where $\chi_{C_{i}}$ is the indicator function for the cell $C_{i}$, and $\Omega_{i}=\operatorname{mes}\left(C_{i}\right)$ represents the volume of cell $C_{i}$. Since the space of all step functions on $\mathbb{M}_{h}$ is isomorphic to $\mathbb{R}^{N}$, we can consider the step functions as vectors. To deal with the step functions as vectors we define a projection $P_{\mathbb{M}_{h}}$ such that:

$$
\left(P_{\mathbb{M}_{h}} \varphi\right)_{i}=\frac{1}{\Omega_{i}} \int_{C_{i}} \varphi .
$$

And to reconstruct a step function from an arbitrary vector $\varphi_{h} \in \mathbb{R}^{N}$ we define an operator $S_{\mathbb{M}_{h}}$ :

$$
S_{\mathbb{M}_{h}} \varphi_{h}(x)=\left(\varphi_{h}\right)_{i}, \quad \text { if } x \in C_{i} .
$$

Thus, the operators $R_{\mathbb{M}_{h}}, P_{\mathbb{M}_{h}}$ and $S_{\mathbb{M}_{h}}$ are related though the following equality:

$$
R_{\mathbb{M}_{h}}=S_{\mathbb{M}_{h}} P_{\mathbb{M}_{h}}
$$

The vector $\varphi_{h}$ will be called a discrete function and it can be considered as an

\footnotetext{
${ }^{1}$ Many dynamical properties of $g$, such as ergodicity, mixing etc., can be studied using spectral properties of $U_{g}$. The idea of using methods of Hibert spaces to study dynamical systems was fist suggested by Koopman in [24] and is usually called koopmanism.
} 
approximation of a continuous function $\varphi$ since when $h \rightarrow 0$,

$$
\left\|S_{\mathbb{M}_{h}} \varphi_{h}-\varphi\right\|_{C^{0}} \rightarrow 0 .
$$

We also introduce a discrete approximation of the $L_{2}$ inner product of functions $\langle\varphi, \psi\rangle=\int_{M} \varphi \psi:$

$$
\left\langle\varphi_{h}, \psi_{h}\right\rangle=\sum_{i} \Omega_{i}\left(\varphi_{h}\right)_{i}\left(\psi_{h}\right)_{i} .
$$

Discrete diffeomorphisms. A matrix $q_{h} \in \mathcal{M}^{N}$ (here $\mathcal{M}^{N}$ is the space of realvalued $N \times N$ matrices) acts on a vector $\varphi_{h}$, therefore we will say that $q_{h}$ approximates $U_{g}$ if $S_{\mathbb{M}_{h}}\left(q_{h} \varphi_{h}\right)$ is close to $U_{g} \varphi$ :

Definition 3. Consider a family of meshes $\mathbb{M}_{h}$ of size $h$, each consisting of $N_{h}$ cells $C_{i}^{h}$. We will say that a family of matrices $q_{h} \in \mathcal{M}^{N}$ approximates a diffeomorphism $g \in \operatorname{SDiff}(M)$ (and denote this property as: $q_{h} \rightsquigarrow g$ ) if the following is true:

$$
S_{\mathbb{M}_{h}}\left(q_{h} P_{\mathbb{M}_{h}} \varphi\right) \stackrel{C^{0}}{\longrightarrow} U_{g} \varphi \quad \text { for every } \varphi \in C(M ; \mathbb{R}) .
$$

In order to better respect the continuous structures at play, we further enforce that our discrete configuration space of diffeomorphisms satisfies two key properties of $U_{g}$ : volume-preservation, reflecting the fact that $U_{g}$ is unitary, and total mass preservation, as $U_{g}$ preserves constants. We will thus only consider matrices $q$ that:

- preserve the discrete $L_{2}$ inner product of functions, i.e.,

$$
\left\langle q \varphi_{h}, q \psi_{h}\right\rangle=\left\langle\varphi_{h}, \psi_{h}\right\rangle
$$

where the inner product of discrete functions is defined by Eq. (2.8). Note that this discrete notion of volume preservation directly implies that for our mesh $\mathbb{M}_{h}$, if we denote

$$
\Omega=\left(\begin{array}{ccc}
\operatorname{mes}\left(C_{1}\right) & \ldots & 0 \\
\vdots & \ddots & \vdots \\
0 & \ldots & \operatorname{mes}\left(C_{N}\right)
\end{array}\right)
$$


then any volume preserving matrix $q$ must satisfy

$$
q^{T} \Omega q=\Omega
$$

The matrix $q$ is thus $\Omega$-orthogonal, restricted to matrices of determinant 1 .

- preserve constant vectors (i.e., vectors having all coordinates equal) as well:

$$
q \mathbf{1}=\mathbf{1}, \quad \text { where: } \mathbf{1}=\left(\begin{array}{l}
1 \\
\vdots \\
1
\end{array}\right) .
$$

The matrix $q$ must thus be signed stochastic as well.

Consequently, the finite-dimensional space of matrices we will use to discretize volume-preserving diffeomorphisms has the following definition:

Definition 4. Let $\mathbb{M}$ be a mesh consisting of cells $C_{i}, i=1, \ldots, N$ and $\Omega$ is the diagonal matrix consisting of volumes of the cells, i.e., $\Omega_{i i}=\operatorname{mes}\left(C_{i}\right)$ and $\Omega_{i j}=0$ when $i \neq j$. We will call a matrix $q \in \mathcal{M}^{N}$ volume-preserving and constant-preserving with respect to the mesh $\mathbb{M}$ if, for all $j$,

$$
\sum_{i} q_{i j}=1
$$

and

$$
q^{T} \Omega q=\Omega
$$

The set of all such $\Omega$-orthogonal, signed stochastic matrices will be denoted $\mathcal{D}(\mathbb{M})$, and will be used as a discretization of the configuration space $\operatorname{SDiff}(M)$.

Our finite-dimensional configuration space $\mathcal{D}(\mathbb{M})$ for fluid dynamics is thus the intersection of two Lie groups: the $\Omega$-orthogonal group, and the group of invertible stochastic matrices; therefore, it is a Lie group. Note that if all cells of $\mathbb{M}$ have the same volume, i.e., $\Omega=\Omega_{0} \mathrm{Id}$, then a matrix $q \in \mathcal{D}(\mathbb{M})$ is orthogonal and the equality (2.11) implies $\sum_{j} q_{i j}=1$. For such meshes (which include regular grids), the 
matrix $q$ is signed doubly-stochastic.

Remark. An alternate, arguably more intuitive way to discretize a diffeomorphism $g \in \operatorname{SDiff}(M)$ on a mesh $\mathbb{M}$ would be to define a matrix $q$ as:

$$
(q(g))_{i j} \equiv \frac{\operatorname{mes}\left(g^{-1}\left(C_{i}\right) \cap C_{j}\right)}{\operatorname{mes}\left(C_{j}\right)}
$$

This discretization also satisfies by definition a discrete preservation of mass and a (different) notion of volume preservation. While it has the added benefit of enforcing that $q$ has no negative terms (therefore respecting the positivity of $U_{g}$ ), the class of matrices it generates is, unfortunately, only a semi-group, which would be an impediment for establishing a variational treatment of fluids. So instead, we take the orthogonal part of this matrix as our configuration (which can be obtained in practice through polar decomposition).

\subsubsection{Discrete velocity field}

Now that we have established a finite-dimensional configuration space $\mathcal{D}(\mathbb{M})$, we can describe its associated Lie algebra, and show that elements of this Lie algebra form a proper discretization of divergence-free vector fields as expected.

Consider a smooth path in the space of volume-preserving diffeomorphisms $g_{t} \in$ $\operatorname{SDiff}(M)$ with $g_{0}=\mathrm{Id}$, and let $q_{h}(t)$ be an approximation of $g_{t}$, i.e., for any piecewise constant function $\varphi_{h}^{0}$ approximating a smooth function $\varphi^{0} \in C^{1}(M, \mathbb{R})$, a discrete version of $\varphi^{0} \circ g_{t}^{-1}=\varphi(t)$ is expressed as:

$$
\varphi_{h}(t)=q_{h}(t) \varphi_{h}^{0}
$$

Assuming $q_{h}(t)$ is smooth in time we define its Eulerian velocity $A_{h}(t)$ to be

$$
A_{h}(t)=\dot{q}_{h}(t) q_{h}^{-1}(t)
$$


thus yielding

$$
\dot{\varphi}_{h}(t)=A_{h}(t) \varphi_{h}(t)
$$

Since $\frac{d}{d t}\left(\varphi^{0} \circ g_{t}^{-1}\right)=-\left\langle\mathbf{d} \varphi(t), v_{t}\right\rangle=-L_{v_{t}} \varphi$, where $v_{t}=\dot{g}_{t}\left(g_{t}^{-1}\right)$ and $L_{v_{t}}$ is the Lie derivative, the matrix $A_{h}(t)$ can be seen as an approximation of the Eulerian velocity field $v_{t}$, which motivates the following definition:

Definition 5. Consider a one-parameter family of volume-preserving diffeomorphisms $g_{t} \in \operatorname{SDiff}(M)$ and the associated time-dependent vector field $v_{t}=\dot{g}_{t}\left(g_{t}^{-1}\right) \in \operatorname{SVect}(M)$. Consider a family of meshes $\mathbb{M}_{h}$ of size $h$ consisting of cells $C_{i}^{h}$ and a projection operator $P_{\mathbb{M}_{h}}: C(M ; \mathbb{R}) \rightarrow \mathbb{R}^{N_{h}}$ defined by $E q$. . (2.4).

We will say that a family of matrices $A_{h}(t) \in \mathcal{M}^{N_{h}}$ approximates a vector field $v_{t}$ (denoted by $A_{h}(t) \rightsquigarrow v_{t}$ ) if the following statement is true:

$$
S_{\mathbb{M}_{h}}\left(A_{h}(t) P_{\mathbb{M}_{h}} \varphi\right) \stackrel{C^{0}}{\longrightarrow}-L_{v_{t}} \varphi \quad \text { for every } \varphi \in C^{\infty}(M ; \mathbb{R}) .
$$

Now, if a matrix $q$ belongs to our configuration space $\mathcal{D}(\mathbb{M})$ (i.e., if $q$ is $\Omega$ orthogonal signed stochastic), then its associated $A$ belongs to its Lie algebra that we will denote as $\mathfrak{D}(\mathbb{M})$. In particular, matrices from this Lie algebra inherit the properties that their columns must sum to zero:

$$
\sum_{i} A_{i j}=0 \quad \text { (preservation of mass), }
$$

and they are $\Omega$-antisymmetric:

$$
A^{T} \Omega+\Omega A=0 \quad \text { (preservation of volume). }
$$

These two properties can be intuitively understood as discrete statements that $A$ represents an advection, and the vector field representing this advection is divergencefree. Lie algebra elements for arbitrary simplicial meshes will be called null-column $\Omega$-antisymmetric matrices. Note that if the mesh is regular $\left(\Omega=\Omega_{0}\right.$ Id), $q$ belongs to the orthogonal group and the matrix $A$ has to be antisymmetric with both its rows 
and columns summing to zero ("doubly null").

Finally, the connection between convergence of $A_{h}(t)$ to $-L_{v_{t}}$ and convergence of $q_{h}(t)$ to $U_{g_{t}}$ is described by the following lemma.

Lemma 3. Consider a one-parameter family of volume-preserving diffeomorphisms $g_{t} \in \operatorname{SDiff}(M)$ and its time-dependent vector field $v_{t}=\dot{g}_{t}\left(g_{t}^{-1}\right) \in \operatorname{SVect}(M)$. Consider a family of meshes $\mathbb{M}_{h}$ of size $h$ consisting of cells $C_{i}^{h}$ and a projection operator $P_{\mathbb{M}_{h}}: C(M ; \mathbb{R}) \rightarrow \mathbb{R}^{N_{h}}$ defined by Eq. (2.4).

Suppose a family of matrices $A_{h}(t) \in \mathfrak{D}\left(\mathbb{M}_{h}\right)$ approximates the Lie derivative $L_{v_{t}}$ in the sense of Definition 5 uniformly in $t$ when $t \in[0, T]$ for some $T>0$.

Then there exists a family of matrices $q_{h}(t) \in \mathcal{D}_{\mathbb{M}_{h}}$ such that $A_{h}(t)=\dot{q}_{h}(t) q_{h}(t)^{-1}$ and $q_{h}(t)$ approximates $g_{t}$ :

$$
S_{\mathbb{M}_{h}}\left(q_{h}(t) P_{\mathbb{M}_{h}} \varphi\right) \stackrel{C^{0}}{\longrightarrow} U_{g_{t}} \varphi \quad \text { for every } \varphi \in C(M ; \mathbb{R}) .
$$

Proof. Consider a family of smooth functions $\varphi(t, x)$ satisfying the advection equation

$$
\dot{\varphi}(t, x)=-L_{v_{t}} \varphi(t, x) .
$$

Suppose that $\tilde{\varphi}_{h}(0, x)=S_{\mathbb{M}_{h}} P_{\mathbb{M}_{h}} \varphi(0, x)$ is an approximation to $\varphi(0, x)$ with:

$$
\sup _{x \in M}\left|\tilde{\varphi}_{h}(0, x)-\varphi(0, x)\right|<\epsilon_{1}
$$

and that $\varphi_{h}(t)=P_{\mathbb{M}_{h}} \tilde{\varphi}_{h}(t, x)$ satisfies the discrete advection equation

$$
\dot{\varphi}_{h}(t, x)=A_{h}(t) \varphi_{t}(t, x) .
$$

Since $A_{h}(t)$ approximates $-L_{v_{t}}$, given $\varepsilon_{2}>0$, we can choose $h$ such that:

$$
\left\|S_{\mathbb{M}_{h}}\left(A_{h}(t) \varphi_{h}(t)\right)+L_{v_{t}} \varphi\right\|<\epsilon_{2}, \quad \text { for all } t \in[0, T] .
$$


Therefore,

$$
\left\|S_{\mathbb{M}_{h}}\left(\dot{\varphi}_{h}(t)\right)(x)-\dot{\varphi}(t, x)\right\|<\epsilon_{2}, \quad \text { for all } t \in[0, T]
$$

and

$$
\left\|S_{\mathbb{M}_{h}}\left(\varphi_{h}(t)\right)(x)-\varphi(t, x)\right\|<\epsilon_{2}+\epsilon_{2} t
$$

So, we have shown that $\varphi_{h}(t, x)$ approximates $\varphi(t, x)$. But $\varphi(t, x)$ satisfies

$$
\varphi(t, x)=U_{g_{t}} \varphi(0, x)
$$

and $\varphi_{h}$ satisfies

$$
\varphi_{h}(t)=q(t) \varphi_{h}(0)
$$

where $q(t)$ is a matrix satisfying the equation

$$
\dot{q}(t)=A_{h}(t) q(t)
$$

Therefore, we see that $q(t) \varphi(0)$ approximates $U_{g_{t}} \varphi(0, x)$. Thus, $A_{h}(t) \rightsquigarrow v_{t}$ implies that $q(t) \rightsquigarrow g_{t}$.

\subsubsection{Discrete commutator}

A discrete flow that approximates a continuous flow $g(t) \in \operatorname{SDiff}(M)$ is defined as a smooth path $q_{h}(t) \in \mathcal{D}(\mathbb{M})$ in the space of $\Omega$-orthogonal signed stochastic matrices, such that $q_{h}(t) \rightsquigarrow g(t) \in \operatorname{SDiff}(M)$ (see Definition 4) and $A_{h}(t)=\dot{q}_{h}(t) q_{h}^{-1}(t) \rightsquigarrow v_{t}=$

$\dot{g}_{t}\left(g_{t}^{-1}\right)$ (see Definition 5). We will now show that the structure of Lie algebra in the space of divergence-free vector fields is preserved by our discretization. Indeed, if two matrices $A$ and $B$ approximate vector fields $u$ and $v$ then their commutator $[A, B]$ approximates the commutator of the Lie derivative operators:

$$
[A, B] \rightarrow L_{u} L_{v}-L_{v} L_{u}
$$


Since $L_{u} L_{v}-L_{v} L_{u}=L_{[u, v]}$, we immediately have

$$
[A, B] \rightsquigarrow-[u, v]
$$

where $[.,$.$] is the commutator of vector-fields or matrices. This property will be useful$ to deal with Lin constraints in our discrete setting.

\subsubsection{Non-holonomic constraints}

For a smooth path $q(t)$, the matrix $A(t)$ describes the infinitesimal exchanges of fluid particles between any pair of cells $C_{i}$ and $C_{j}$. It is thus natural to assume that $A_{i j}$ is non-zero only if cells $C_{i}$ and $C_{j}$ share a common boundary, i.e., are immediate neighbors. This sparsity will be numerically advantageous later on to reduce the computational complexity of the resulting integration schemes. We thus choose to restrict discrete paths $\{q(t)\}$ on $\mathcal{D}(\mathbb{M})$ to those for which $A(t)$ satisfies these constraints. In other words, we only consider column-null $\Omega$-antisymmetric matrices satisfying the constraints as valid discrete vector fields. The non-zero elements of these matrices correspond to boundaries between adjacent cells, and can be interpreted as directional transfer densities (per second) from $C_{i}$ to $C_{j}$ - they could abusively be called "fluxes" on regular grids; but we will make the proper link with the integrals of the velocity field over mesh faces later on.

More formally, we define the constrained set $S_{q} \subset T_{q} \mathcal{D}(\mathbb{M})$ as the set of matrices corresponding to exchanges between neighboring cells only, i.e., $\dot{q} \in S_{q}$ if and only if $\left(\dot{q} q^{-1}\right)_{i j} \neq 0$ implies that the cells $C_{i}$ and $C_{j}$ are neighbors. In this case the matrix $A$ is defined by a set of non-zero values $A_{i j}$ defined on walls between adjacent cells $C_{i}$ and $C_{j}$. To indicate their adjacency, we will write that $j \in N(i)$ and $i \in N(j)$, where $N(k)$ refers to the set of adjacent cells to cell $C_{k}$ in the mesh $\mathbb{M}$. We will say that a matrix $A$ belongs to the class $\mathcal{N}$ if $A_{i j} \neq 0$ implies $j \in N(i)$. Finally, we will denote by $\mathcal{S} \equiv S_{\text {Id }}=\mathfrak{D}(\mathbb{M}) \cap \mathcal{N}$ the constrained set at identity. Consequently, our dynamic treatment of fluid motion will only consider matrices $A$ in $\mathcal{S} \subset \mathcal{D}(\mathbb{M})$, i.e., matrices in $\mathcal{D}(\mathbb{M})$ satisfying the constraints. 
Note that if two matrices $A$ and $B$ both satisfy the constraints, their commutator does not (unless $[A, B]=0$ ): while the element of the commutator corresponding to any pair of cells which are more than two cells away is zero, the element $[A, B]_{i j}$ may be non-zero when cells $C_{i}$ and $C_{j}$ are "two-cells away" from each other since

$$
[A, B]_{i j}=\sum_{k}\left(A_{i k} B_{k j}-B_{i k} A_{k j}\right)
$$

Notice that the commutator is zero for neighboring cells as well since $A_{k k}=B_{k k}=0$ due to their $\Omega$-antisymmetry. So, we see that if $[\mathcal{S}, \mathcal{S}]=\{[A, B] \mid A, B \in \mathcal{S}\}$ then $\mathcal{S} \cap[\mathcal{S}, \mathcal{S}]=\{\mathbf{0}\}$, where $\mathbf{0}$ is the zero matrix. Therefore, the constraints we just defined are non-holonomic.

Remark. When a discrete vector field $A$ is in the set $\mathcal{S}$, the non-zero values $\Omega_{i} A_{i j}$ of the antisymmetric matrix $\Omega A$ can be understood as dual 1-chains, i.e., 1-dimensional chains on the dual of $\mathbb{M}$ [31]. This connection with 1-chains will become crucial later when dealing with advection of curves to derive a discrete Kelvin's theorem in Section 2.3.2.

\subsubsection{Relation between $A$ and Fluxes}

Suppose we have a family of discrete flows $q^{h}(t)$ which approximates a flow $g_{t} \in$ $\operatorname{SDiff}(M)$ such that $A_{h}(t)=\dot{q}_{h}(t) q_{h}(t)^{-1}$ approximates $L_{v_{t}}$ and satisfies the NHC. Let's see how individual elements $\left(A_{h}\right)_{i j}(t)$ of $A_{h}(t)$ can be considered as an approximation to values of $v_{t}(x)$ for some $x \in M$. Recall that

$$
\dot{\varphi}_{h}(t)=A_{h}(t) \varphi_{h}(t)
$$

is a discrete version of the advection equation

$$
\dot{\varphi}=-L_{v_{t}} \varphi
$$


and $A_{h}(t) \varphi_{h}(t) \rightarrow-L_{v_{t}} \varphi$ in $C^{0}$ norm. But it also means that $\left(\Omega A_{h}(t) \varphi_{h}(t)\right)_{i}$ is an approximation to the integral $-\int_{C_{i}} L_{v_{t}} \varphi_{t}$, i.e.,

$$
\sum_{j \in N(i)} \Omega_{i}\left(A_{h}\right)_{i j}(t) \varphi_{j}(t) \approx-\int_{C_{i}} L_{v_{t}} \varphi_{t} \stackrel{\nabla \cdot v_{t}=0}{=}-\int_{\partial C_{i}} \varphi_{t}(v, \vec{n})
$$

where $\vec{n}$ is the normal vector to the boundary of $C_{i}$. Therefore, an element $\Omega_{i}\left(A_{h}\right)_{i j}(t)$ can be considered as an approximation to the flux of vector field $v(t)$ through the boundary $D_{i j}$ between the cells $C_{i}$ and $C_{j}$ (with $\vec{n}_{i j}$ the normal vector to $D_{i j}$ oriented from $C_{i}$ to $\left.C_{j}\right)$ :

$$
\Omega_{i} A_{i j}(t) \approx-\int_{D_{i j}}\left(v, \vec{n}_{i j}\right)
$$

We know that $\int_{D_{i j}}(v, \vec{n}) \approx\left(v_{t}\left(x_{i j}\right), \vec{n}_{i j}\right) S_{i j}+O\left(h^{2}\right)$, where $x_{i j}$ is the barycenter of the boundary $D_{i j}$ and $S_{i j}$ is the area of $D_{i j}$. Therefore, we obtain that, up to a sign, $\Omega_{i}\left(A_{h}\right)_{i j}$ approximates the flux through the boundary between $C_{i}$ and $C_{j}$ :

$$
\left(A_{h}\right)_{i j}(t) \approx-\left(v_{t}\left(x_{i j}\right), \vec{n}_{i j}\right) \frac{S_{i j}}{\Omega_{i}}
$$

In case of a Cartesian grid of size $h$ this formula simplifies to:

$$
\left(A_{h}\right)_{i j}(t) \approx-\frac{\left(v_{t}\left(x_{i j}\right), \vec{n}_{i j}\right)}{h}
$$

\subsubsection{Lagrangian dynamics with non-holonomic constraints on $\mathcal{D}(\mathcal{M})$}

The overall goal of this thesis is to approximate geodesic flows on $\operatorname{SDiff}(M)$ by Lagrangian flows on $\mathcal{D}(\mathbb{M})$. To achieve this goal, we first need to define a Lagrangian $L_{h}(\dot{q}, q)$ such that

$$
L_{h}(\dot{q}, q) \rightarrow \int_{M} \frac{1}{2} v^{2} \quad \text { when } \dot{q} q^{-1} \rightsquigarrow v
$$

and

$$
\delta L_{h}(\dot{q}, q) \rightarrow \delta \int_{M} \frac{1}{2} v^{2} \quad \text { when } \dot{q} q^{-1} \rightsquigarrow v \text { and } \delta\left(\dot{q} q^{-1}\right) \rightsquigarrow \delta v
$$


For this Lagrangian we can formulate the Lagrange-d'Alembert principle with nonholonomic constraints:

$$
\delta \int_{0}^{1} L_{h}(q, \dot{q}) d t=0 \text { with }\left\{\begin{array}{l}
\delta q \in S_{q} \\
\delta q(0)=\delta q(1)=0
\end{array}\right.
$$

Suppose $L_{h}$ depends only on $A=\dot{q} q^{-1}$. It can be shown that $\delta A$ satisfies a discrete version of Lin constraints (see page 41):

$$
\delta A=\dot{B}-[A, B]
$$

where $B=\delta q q^{-1}$. Let's denote by $\frac{\partial L_{h}}{\partial A}$ a matrix which has $\frac{\partial L_{h}}{\partial A_{i j}}$ as its $(i, j)$-element. Then we have

$$
\delta \int_{0}^{1} L_{h}(q, \dot{q}) d t=\int_{0}^{1} \operatorname{Tr}\left(\left(\frac{\partial L_{h}}{\partial A}\right)^{T} \delta A\right) d t=\int_{0}^{1} \operatorname{Tr}\left(\left(\frac{\partial L_{h}}{\partial A}\right)^{T}(\dot{B}-[A, B])\right) d t
$$

We can use equality $\operatorname{Tr}\left(M_{1}\left[M_{2}, M_{3}\right]\right)=\operatorname{Tr}\left(\left[M_{1}, M_{2}\right] M_{3}\right)$ and integration by parts to write:

$$
\delta \int_{0}^{1} L_{h}(q, \dot{q}) d t=-\int_{0}^{1} \operatorname{Tr}\left(\left\{\frac{d}{d t}\left(\frac{\partial L_{h}}{\partial A}\right)^{T}+\left[\left(\frac{\partial L_{h}}{\partial A}\right)^{T}, A\right]\right\} B\right) .
$$

Since condition $\delta q \in S_{q}$ means that $B \in \mathcal{S}$, the Lagrange-d'Alembert principle can be rewritten as

$$
\operatorname{Tr}\left(\left\{\frac{d}{d t}\left(\frac{\partial L_{h}}{\partial A}\right)^{T}+\left[\left(\frac{\partial L_{h}}{\partial A}\right)^{T}, A\right]\right\} B\right)=0, \quad \text { for every } B \in \mathcal{S} .
$$

In order for this equation to be an approximation to the Euler equation, conditions (2.27) and (2.28) must be satisfied. Condition (2.28) implies that

$$
\operatorname{Tr}\left(\left(\frac{\partial L_{h}}{\partial A}\right)^{T}(\dot{B}-[A, B])\right)
$$


must be an approximation to

$$
\langle v, \delta v\rangle=\langle v, \dot{\xi}+[v, \xi]\rangle
$$

when $A \rightsquigarrow v, B \rightsquigarrow \xi$ and $\dot{B} \rightsquigarrow \dot{\xi}$.

Since both $A$ and $B$ belong to the constrained set $\mathcal{S}$, their commutator $[A, B]$ has zero elements for neighboring $i, j:[A, B]_{i j}=0$ for all $i \in N(j)$. Thus, if $\frac{\partial L_{h}}{\partial A}$ belongs to the set $\mathcal{S}$, we have

$$
\operatorname{Tr}\left(\left(\frac{\partial L_{h}}{\partial A}\right)^{T}(\dot{B}-[A, B])\right)=\operatorname{Tr}\left(\left(\frac{\partial L_{h}}{\partial A}\right)^{T} \dot{B}\right),
$$

which cannot be an approximation to $\langle v, \delta v\rangle$. Therefore, we see that the Lagrangian must depend on values $A_{i j}$ where $i \notin N(j)$.

To find a Lagrangian satisfying properties (2.27) and (2.28) let's recall that for vector fields $u$ and $v$ their inner product $(u, v)$ can be written as a pairing of $v$ with a 1 -form $u^{b}$ :

$$
(u, v)=\left\langle u^{b}, v\right\rangle .
$$

Thus, we will look for a Lagrangian $L_{h}$ having the form

$$
L_{h}(A)=\frac{1}{4} \operatorname{Tr}\left(\Omega A\left(A^{b}\right)^{T}\right),
$$

where the flat operator $b$ will be defined to satisfy the property that

$$
\frac{1}{2} \operatorname{Tr}\left(\Omega B\left(A^{b}\right)^{T}\right) \rightarrow \int_{M}(u, v), \quad \text { when } A \rightsquigarrow u, B \rightsquigarrow v
$$

where $A \in \mathcal{S}$ and $B \in \mathcal{S}$ and

$$
\frac{1}{2} \operatorname{Tr}\left(\Omega B\left(A^{b}\right)^{T}\right) \rightarrow-2 \int_{M}(u, v), \quad \text { when } A \rightsquigarrow u, B \rightsquigarrow v
$$

when $A \in \mathcal{S}$ and $B \in[\mathcal{S}, \mathcal{S}]$. This will guarantee that conditions (2.27) and (2.28) are satisfied (see page 42 for a more detailed explanation). 
In the next section we will present a discretization of differential forms and a few operators acting on them to help us construct $A^{b}$.

\section{$2.2 \quad$ Structure-Preserving Spatial Field Discretiza- tion}

We now introduce a discrete calculus consistent with our discretization of vector fields. Unlike previous discrete exterior calculus approaches, mostly based on chains and

cochains $[14,6,3]$, we clearly distinguish between discrete vector fields and discrete forms acting on them. Moreover, our notion of forms will need to act not only on vector fields satisfying the NHC (being thus very reminiscent of the chain/cochain approach), but also on vector fields having form of a commutator, as imposed by Lin constraints. We also introduce a discrete contraction operator $\mathbf{i}_{v}$ and a discrete Lie derivative to complete our set of spatial operators - we will later show that the algebraic definition of our Lie derivative matches its dynamic counterpart as expected. We will not make any distinction in symbols between the discrete and continuous exterior calculus operators $\left(\mathbf{i}_{v}, \mathbf{d}, L_{v}\right.$, etc) as context will disambiguate their meaning.

\subsubsection{Discrete zero-forms}

In our context, a discrete 0 -form is a function ${ }^{0} \mathrm{~F}$ that is piecewise constant per cell as previously defined in Section 2.1. Note that its representation is a vector of $N$ cell values,

$$
{ }^{0} F=\left({ }^{0} F_{1},{ }^{0} F_{2}, \ldots,{ }^{0} F_{N}\right)^{T}
$$

where ${ }^{0} F_{i}$ represents the value of the function ${ }^{0} F$ in cell $C_{i}$.

Remark Our definition of 0 -forms is no different from dual 0-cochains in dimension $n$ as used extensively in $[31,14]$. They naturally pair with dual 0-chains (i.e., linear combinations of cell centers). 


\subsubsection{Discrete one-forms}

As the space $\mathcal{D}(\mathbb{M})$ of matrices is used to discretize vector fields, a natural way to discretize one-forms is to also use matrices to respect the duality between these two entities. Moreover, it is in line with the previous definition for 0 -forms that were encoded as a 1-tensor: 1-forms will now be encoded by a 2-tensor. Notice that this is also reminiscent of the approximation $T M \approx M \times M$ used in discrete mechanics.

Discrete contraction We define the contraction operator by a discrete vector field $A$, acting on a discrete one-form ${ }^{1} F$ to return a discrete zero-form, as:

$$
\mathbf{i}_{A}{ }^{1} F \equiv \operatorname{diag}\left({ }^{1} F A^{T}\right) \stackrel{\text { def }}{=}\left(\left({ }^{1} F A^{T}\right)_{11}, \ldots,\left({ }^{1} F A^{T}\right)_{N N}\right)^{T}
$$

Notice the metric-independence of this definition and that if the discrete vector field contains only non-zero terms for neighboring cells, any term $\left({ }^{1} F\right)_{i j}$ where cell $C_{i}$ and cell $C_{j}$ are not neighbors does not contribute to the contraction. In this case, the value of the resulting 0 -form for cell $C_{i}$ is thus: $\left(\mathbf{i}_{A}{ }^{1} F\right)_{i}=\sum_{j \in N(i)}{ }^{1} F_{i j} A_{i j}$, which is a local sum of the natural pairings of ${ }^{1} F$ and $A$ on each face of cell $C_{i}$.

Discrete total pairing With this contraction defined, we derive a total pairing between a 1-form and a vector field as:

$$
\left\langle\left\langle{ }^{1} F, A\right\rangle \equiv \frac{1}{2} \operatorname{Tr}\left(\Omega A^{1} F^{T}\right) .\right.
$$

This definition satisfies the following connection with the previously-defined contraction: for all $A \in \mathfrak{D}(\mathbb{M})$

$$
\int_{M} \mathbf{i}_{A}{ }^{1} F d V=\left\langle\left\langle{ }^{1} F, A\right\rangle\right\rangle
$$

Note that the volume form $\Omega$ is now needed to integrate the piecewise-constant 0 form $\mathbf{i}_{A}{ }^{1} F$ over the entire domain. Finally, since the matrix $\Omega A$ is antisymmetric, the symmetric component of ${ }^{1} F$ does not play any role in the pairing.

Therefore, we will assume thereafter that a discrete one-form ${ }^{1} F$ is defined by an 
antisymmetric matrix: ${ }^{1} F \in \mathfrak{s o}(N)$.

Remark When viewed as acting on vector fields in the NHC $\mathcal{S}$, our representation of discrete 1-forms coincides with the use of 1-cochains on the dual of $\mathbb{M}[14]$ : the value ${ }^{1} F_{i j}$ (resp., ${ }^{1} F_{j i}$ ) can be understood as the integral of a continuous 1 -form ${ }^{1} f$ on the dual edge linking cells $C_{i}$ and $C_{j}$ (resp., $C_{j}$ and $C_{i}$ ). However, our use of antisymmetric matrices extends this cochain interpretation to arbitrary discrete vector fields. This will become particularly useful when 1-forms need to be paired with vector fields issued from the commutator $[A, B]$ of two vector fields $A$ and $B$ both in $\mathcal{S}$, as hinted in Section 2.1.6.

\subsubsection{Discrete two-forms}

We extend our definition of one-forms to two-forms in a similar fashion: discrete 2forms will be encoded as 3 -tensors ${ }^{3} F_{i j k}$ that are antisymmetric w.r.t. the last two indices.

Discrete contraction Contraction of a 2 -form ${ }^{2} F$ by a vector field $A$ is defined as:

$$
\left(\mathbf{i}_{A}{ }^{2} F\right)_{i j}=\frac{1}{2} \sum_{k}\left({ }^{2} F_{i j k} A_{i k}-{ }^{2} F_{j i k} A_{j k}\right) .
$$

Notice again here that the resulting discrete 1-form is indeed an antisymmetric matrix (by construction), and that if $A \in \mathcal{S}$, many of the terms in the sum vanish.

Discrete total pairing The total pairing of a discrete 2 -form ${ }^{2} F$ by two discrete vector fields $A$ and $B$, the discrete equivalent of $\int_{M}{ }^{2} f(a, b) d V$, is now defined as:

$$
\left.\left\langle{ }^{2} F,(A, B)\right\rangle\right\rangle \equiv \frac{1}{2} \sum_{i, j, k} \Omega_{i}{ }^{2} F_{i j k} A_{i k} B_{i j} .
$$


This definition satisfies the expected property linking contraction and pairing: for all $B \in \mathfrak{D}$

$$
\left\langle\left\langle\mathbf{i}_{A}^{2} F, B\right\rangle\right\rangle=\left\langle\left\langle{ }^{2} F,(A, B)\right\rangle\right\rangle
$$

It also is compatible with our former definitions, since:

$$
\begin{aligned}
\int \mathbf{i}_{B}\left(\mathbf{i}_{A}{ }^{2} F\right) d V & =\frac{1}{2} \sum_{i}\left(\frac{1}{2} \sum_{j, k}\left(\Omega_{i}{ }^{2} F_{i j k} A_{i k}-\Omega_{i}{ }^{2} F_{j i k} A_{j k}\right) B_{i j}\right) \\
& =\frac{1}{2} \sum_{i}\left(\frac{1}{2} \sum_{j, k}\left(\Omega_{i}{ }^{2} F_{i j k} A_{i k} B_{i j}+\Omega_{j}{ }^{2} F_{j i k} A_{j k} B_{j i}\right)\right) \\
& =\frac{1}{2} \sum_{i}\left(\sum_{j, k} \Omega_{i}{ }^{2} F_{i j k} A_{i k} B_{i j}\right) \\
& =\left\langle\left\langle\mathbf{i}_{A}{ }^{2} F, B\right\rangle .\right.
\end{aligned}
$$

\subsubsection{Other operators on discrete forms}

A few more operators acting on 0-, 1-, or 2-forms will be valuable to our discretization of incompressible fluids.

Discrete exterior derivative We can define a discrete version $\mathbf{d}$ of the exterior derivative. For a discrete 0 -form ${ }^{0} F$, the one-form $\mathbf{d}^{0} F$ is formally defined as

$$
\left(\mathbf{d}^{0} F\right)_{i j}={ }^{0} F_{i}-{ }^{0} F_{j} .
$$

Similarly, if ${ }^{1} F$ is a discrete one-form then we can define:

$$
\left(\mathbf{d}^{1} F\right)_{i j k}={ }^{1} F_{i j}+{ }^{1} F_{j k}+{ }^{1} F_{k i} .
$$

More generally, we define our operator $\mathbf{d}$ as acting on a $k$-form ${ }^{k} F$ with:

$$
\left(\mathbf{d}^{k} F\right)_{i_{1} i_{2} \ldots i_{k+1}}=\sum_{j \in[1 . . k+1]}(-1)^{j+1 k} F_{i_{1} \ldots \hat{i_{j} \ldots i_{k+1}}}
$$


where $\widehat{\cdot}$ indicates the omission of a term. This expression respects the antisymmetry of our discrete form representation.

Remark Notice here again that when the centers of cells $C_{i_{1}}, C_{i_{2}}, \ldots, C_{i_{k+1}}$ form a $k$ simplex on the dual of mesh $\mathbb{M}$, our definition of $\mathbf{d}$ simply enforces Stokes' theorem and thus coincides with the discrete exterior derivative widely used in the literature [14]. Our discrete exterior derivative extends this simple geometric property to arbitrary $(k+1)$-tuples of cells.

Discrete Lie derivative Now that we have defined contraction and derivatives on discrete one-forms we can define the Lie derivative using Cartan's "magic" formula in the continuous setting

$$
L_{v}=\mathbf{i}_{v} \mathbf{d}+\mathbf{d} \mathbf{i}_{v}
$$

Definition 6. Let $A$ be a discrete vector field satisfying the $N H C$ and ${ }^{1} F$ be an antisymmetric discrete one-form. Then the Lie derivative of ${ }^{1} F$ along $A$ is defined as

$$
L_{A}{ }^{1} F=\mathbf{i}_{A} \mathbf{d}^{1} F+\mathbf{d i}_{A}{ }^{1} F .
$$

Lemma 4. For a vector field represented through an $\Omega$-antisymmetric and columnnull $A$, and a discrete closed one-form represented as a column-null and antisymmetric ${ }^{1} F$ :

$$
\left(L_{A}{ }^{1} F\right)_{i j}=\frac{1}{2}\left(\left[{ }^{1} F \Omega, A\right] \Omega^{-1}\right)_{i j}+\frac{1}{2}\left(\left({ }^{1} F A^{T}\right)_{i i}-\left({ }^{1} F A^{T}\right)_{j j}\right) .
$$

Proof. As $A$ is $\Omega$-antisymmetric and column-null, we have

$$
\sum_{k}{ }^{1} F_{i j} A_{i k}={ }^{1} F_{i j} \sum_{k} A_{i k}=0
$$


Therefore,

$$
\begin{aligned}
\left(\mathbf{i}_{A} \mathbf{d}^{1} F\right)_{i j}= & \frac{1}{2} \sum_{k}\left(\left(\mathbf{d}^{1} F\right)_{i j k} A_{i k}-\right. \\
=\frac{1}{2} \sum_{k}\left({ }^{1} F_{i j} F\right)_{j i k} A_{j k} F_{j k}+ & \left.{ }^{1} F_{k i}\right) A_{i k}-\frac{1}{2} \sum_{k}\left({ }^{1} F_{j i}+{ }^{1} F_{i k}+{ }^{1} F_{k j}\right) A_{j k} \\
& =-\frac{1}{2}\left(\left(A^{1} F\right)_{i j}+\left({ }^{1} F A^{T}\right)_{i i}-\left(A^{1} F\right)_{j i}-\left({ }^{1} F A^{T}\right)_{j j}\right) .
\end{aligned}
$$

Now, since $A^{T}=-\Omega A \Omega^{-1}$ and ${ }^{1} F^{T}=-{ }^{1} F$, we can write:

$$
\left(A^{1} F\right)_{j i}=\left(\left(A^{1} F\right)^{T}\right)_{i j}=\left({ }^{1} F \Omega A \Omega^{-1}\right)_{i j}
$$

and, therefore,

$$
\left(\mathbf{i}_{A} \mathbf{d}^{1} F\right)_{i j}=\frac{1}{2}\left(\left(\left[{ }^{1} F \Omega, A\right] \Omega^{-1}\right)_{i j}+\left({ }^{1} F A^{T}\right)_{j j}-\left({ }^{1} F A^{T}\right)_{i i}\right) .
$$

Also, one has

$$
\mathbf{i}_{A}{ }^{1} F=\operatorname{diag}\left({ }^{1} F A^{T}\right),
$$

therefore,

$$
\left(\operatorname{di}_{A}{ }^{1} F\right)_{i j}=\left({ }^{1} F A^{T}\right)_{i i}-\left({ }^{1} F A^{T}\right)_{j j},
$$

which implies the result.

This formula can be seen as a discrete version of the relation between Lie derivative and covariant derivative in the continuous case:

$$
L_{v} v^{b}=\left(\nabla_{v} v\right)^{b}+\frac{1}{2} \mathbf{d}\left\langle v^{b}, v\right\rangle .
$$

\subsubsection{Discrete flat operator}

Recall that the continuous flat of a vector field $v$ is a 1 -form $v^{b}$ such that

$$
\left\langle v^{b}, w\right\rangle=(v, w), \quad \text { for every vector field } w,
$$


where $(v, w)$ is the inner product of vector fields. Discretizing this inner product for vector fields represented as column-null $\Omega$-antisymmetric matrices is far from obvious. Instead, we propose to define a discrete flat operator $b_{h}: A \mapsto A^{b_{h}}$ such that the pairing of matrices $\left\langle\left\langle A^{b_{h}}, B\right\rangle\right\rangle=\frac{1}{2} \operatorname{Tr}\left(\Omega A^{b_{h}} B^{T}\right)$ approximates the product of vector fields integrated on $M$ :

$$
\left\langle\left\langle A^{b_{h}}, B\right\rangle\right\rangle=\frac{1}{2} \operatorname{Tr}\left(\Omega A^{b_{h}} B^{T}\right) \rightarrow \int_{M}(v(x), w(x)) d x, \quad \text { when } h \rightarrow 0
$$

if $A \rightarrow L_{v}$ and $B \rightarrow L_{w}$. Note that the multiplication by $\Omega$ simply integrates the 0-form over each cell, thus we define the integrated product as $\operatorname{Tr}\left(\Omega A^{b_{h}} B^{T}\right)$ while the cell-wise product is $\operatorname{diag}\left(A^{b_{h}} B^{T}\right)$.

Note that the operator $b_{h}$ cannot be trivial. Indeed, for any matrices $A, B, C \in \mathcal{S}$, we have $\operatorname{Tr}(A[B, C])=0$ because $\mathcal{S} \cap[\mathcal{S}, \mathcal{S}]=\{\mathbf{0}\}$; but we can choose $A, B$ and $C$ to approximate vector fields $v, u$ and $w$ such that $\int_{M}(v,[u, w]) \neq 0$. The problem here is that the matrix $A$ can store more information than necessary to encode a vector field. To resolve this issue we will consider only matrices satisfying the non-holonomic constraints and matrices resulting from the commutator of two matrices satisfying the non-holonomic constraints to define our discrete flat operator.

Definition 7. Consider a family of meshes $\mathbb{M}_{h}$ of size $h$. An operator

$$
b_{h}: \mathcal{S} \rightarrow \mathfrak{D}\left(\mathbb{M}_{h}\right)
$$

is called a discrete flat operator if the following two conditions are satisfied:

$$
\begin{aligned}
& \left\langle\left\langle A_{h}^{b_{h}}, B_{h}\right\rangle\right\rangle \rightarrow \int_{M}(v(x), u(x)) d x, \quad \text { when } h \rightarrow 0, \\
& \quad \text { for every } A_{h}, B_{h} \in \mathcal{S}, A_{h} \rightarrow-L_{v}, B_{h} \rightarrow-L_{u}
\end{aligned}
$$




$$
\begin{aligned}
\left\langle\left\langle A_{h}^{b_{h}},\left[B_{h}, C_{h}\right]\right\rangle\right\rangle \rightarrow-2 \int_{M}(v(x),[u, w](x)) d x, \quad \text { when } h \rightarrow 0 \\
\quad \text { for every } A_{h}, B_{h}, C_{h} \in \mathcal{S}, A_{h} \rightarrow-L_{v}, B_{h} \rightarrow-L_{u}, C_{h} \rightarrow-L_{w} .
\end{aligned}
$$

Note that in this definition, $\left\langle\left\langle A^{b}, X\right\rangle\right\rangle$ approximates the inner product when $X \in \mathcal{S}$ and twice the inner product when $X \in[\mathcal{S}, \mathcal{S}]$. In essence, an element of $[\mathcal{S}, \mathcal{S}]$ represents twice a velocity; see the remark on page 42 for a detailed explanation.

The next lemma gives a necessary and sufficient condition for a discrete flat operator:

Lemma 5. A family of operators $b_{h}$ satisfies condition (2.44) if and only if for every $A_{h}, B_{h}, C_{h} \in \mathcal{S}$ approximating vector fields $v, u, w \in \operatorname{SVect}(M)$ respectively we have

$$
\left\langle\left\langle d A_{h}^{b_{h}},\left(B_{h}, C_{h}\right)\right\rangle\right\rangle \rightarrow \int_{M} \omega(u, w) d V, \quad \text { where } \omega=d v^{b}
$$

Proof. First, let's show that for any $u, v, w \in \operatorname{SVect}(M)$

$$
\int_{M}(v(x),[u, w](x)) d x=\int_{M} \mathbf{d} v^{b}(u, w) d x
$$

Indeed, since

$$
\int_{M}(v(x),[u, w](x)) d x=\int_{M} \mathbf{i}_{[u, w]} v^{b}
$$

and (see [25])

$$
\mathbf{i}_{[u, w]} v^{b}=L_{u} \mathbf{i}_{w} v^{b}-\mathbf{i}_{w} L_{u} v^{b}
$$

we have

$$
\int_{M}(v(x),[u, w](x)) d x=\int_{M} L_{u} \mathbf{i}_{w} v^{b}-\mathbf{i}_{w} L_{u} v^{b} \stackrel{\nabla \cdot u=0}{=}-\int_{M} \mathbf{i}_{w} L_{u} v^{b}
$$

But, by Cartan's formula $L_{u} v^{b}=\mathbf{i}_{u} \mathbf{d} v^{b}+\mathbf{d i}_{u} v^{b}$. Therefore,

$$
\int_{M} \mathbf{i}_{w} L_{u} v^{b}=\int_{M} \mathbf{i}_{w} \mathbf{i}_{u} \mathbf{d} v^{b}=\int_{M} \mathbf{d} v^{b}(u, w)
$$

where we used the fact that $w \in \operatorname{SVect}(M)$ and therefore $\int_{M} \mathbf{i}_{w} \mathbf{d i}_{u} v^{b}=0$. 


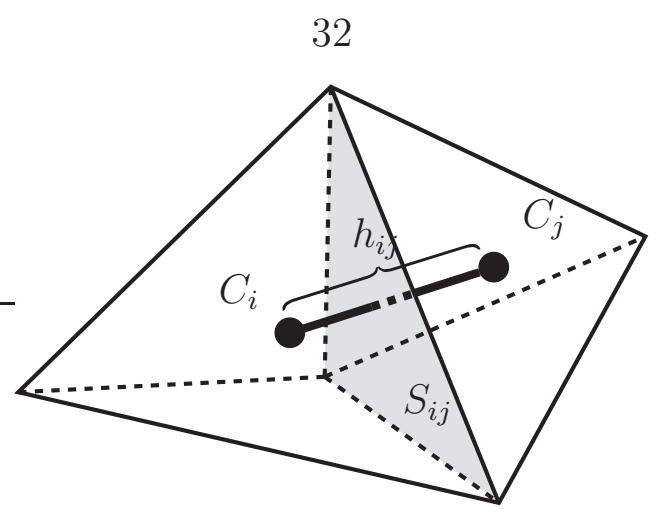

Figure 2.1: Spatial Discretization: two cells $C_{i}$ and $C_{j}$, along with their common face $D_{i j}$ of area $S_{i j}$ and its dual edge $e_{i j}$ of length $h_{i j}$.

Now, let's show that

$$
2\left\langle\left\langle\mathbf{d} A^{b},(B, C)\right\rangle\right\rangle=-\left\langle\left\langle A^{b},[B, C]\right\rangle\right\rangle .
$$

Using properties of the trace operator we get

$$
\left\langle\left\langle A^{b},[B, C]\right\rangle\right\rangle=\frac{1}{2} \operatorname{Tr}\left(\Omega A^{b}[B, C]^{T}\right)=\frac{1}{2} \operatorname{Tr}\left(A^{b} \Omega[B, C]\right)=\frac{1}{2} \operatorname{Tr}\left(\left[A^{b} \Omega, B\right] C\right) .
$$

By Lemma $4,\left[A^{b} \Omega, B\right]=2\left(L_{B} A^{b}\right) \Omega+2 \mathbf{d i}_{B} A^{b} \Omega$. Thus,

$$
\frac{1}{2} \operatorname{Tr}\left(\left[A^{b} \Omega, B\right] C\right)=\operatorname{Tr}\left(\mathbf{i}_{B} \mathbf{d} A^{b} \Omega C\right)=-2\left\langle\left\langle\mathbf{d} A^{b},(B, C)\right\rangle\right\rangle .
$$

Construction of a flat operator on a simplicial mesh. Now we show how to construct a flat operator $b: A \mapsto A^{b}$ on a two-dimensional simplicial mesh. Construction on an arbitrary mesh in the two- or three-dimensional case is similar. Let's recall that $A$ is assumed to satisfy the non-holonomic constraints, i.e., $A \in \mathcal{S}$.

First, the flat operator should satisfy the following property:

$$
\left\langle\left\langle A_{h}^{b_{h}}, B_{h}\right\rangle\right\rangle \underset{h \rightarrow 0}{\longrightarrow} \int_{M}(v(x), u(x)) d x \text { for } A \underset{h \rightarrow 0}{\rightsquigarrow} v \text { and } B \underset{h \rightarrow 0}{\rightsquigarrow} u \text {. }
$$

Since $B_{h} \in \mathcal{S}$, only terms $\left(A_{h}^{b_{h}}\right)_{i j}$ with $j \in N(i)$ appear in the expression $\left\langle\left\langle A_{h}^{b_{h}}, B_{h}\right\rangle\right\rangle$ 
above. Therefore, these terms can be thought of as assigned to the dual edges connecting cells $C_{i}$ and $C_{j}$ (see Figure 2.1). Define $A^{b}$ on these edges by

$$
A_{i j}^{b}=A_{i j} \Omega_{i} \frac{h_{i j}}{S_{i j}}, \quad \text { when } j \in N(i) .
$$

As in Section 2.1.5, $\Omega_{i} A_{i j}$ is an approximation to the flux of vector field through the boundary between cells $C_{i}$ and $C_{j}$, i.e.,

$$
\Omega_{i} A_{i j} \approx-\left(v\left(x_{i j}\right), \vec{n}_{i j}\right) S_{i j},
$$

where $x_{i j}$ is the barycenter of boundary between cells $C_{i}$ and $C_{j}$, and $\vec{n}_{i j}$ is the normal to this boundary, oriented from $C_{i}$ to $C_{j}$. Thus, $A^{\text {b }}$ can be written as

$$
A_{i j}^{b} \approx-\left(v\left(x_{i j}\right), \vec{n}_{i j}\right) h_{i j} \approx \int_{e_{j i}} v^{b},
$$

where $e_{j i}$ is the dual edge connecting cells $C_{i}$ and $C_{j}$. Therefore, $A_{i j}^{b}$ is indeed an approximation to $v^{b}$ integrated along $e_{j i}$ and thus can be considered as a discrete 1-form in the sense of DEC.

Now, the pairing

$$
\left\langle\left\langle A^{b}, B\right\rangle\right\rangle=\frac{1}{2} \operatorname{Tr}\left(\Omega A^{\mathrm{b}} B^{T}\right)
$$

can be written as

$$
\left\langle\left\langle A^{b}, B\right\rangle\right\rangle \approx \frac{1}{2} \sum_{i, j}\left[\left(v\left(x_{i j}\right), \vec{n}_{i j}\right) h_{i j}\right]\left[\left(u\left(x_{i j}\right), \vec{n}\right) S_{i j}\right] .
$$

But $A_{i j}^{b} \approx-\left(v\left(x_{i j}\right), \vec{n}_{i j}\right) h_{i j} \approx \int_{e_{j i}} v^{b}$ and

$$
\left(u\left(x_{i j}\right), \vec{n}\right) S_{i j} \approx \int_{D_{i j}}\left(u, \vec{n}_{i j}\right)=\int_{D_{i j}} * u^{b},
$$

where $D_{i j}$ is the boundary between $C_{i}$ and $C_{j}$ and $\vec{n}_{i j}$ its normal oriented from $C_{i}$ to 


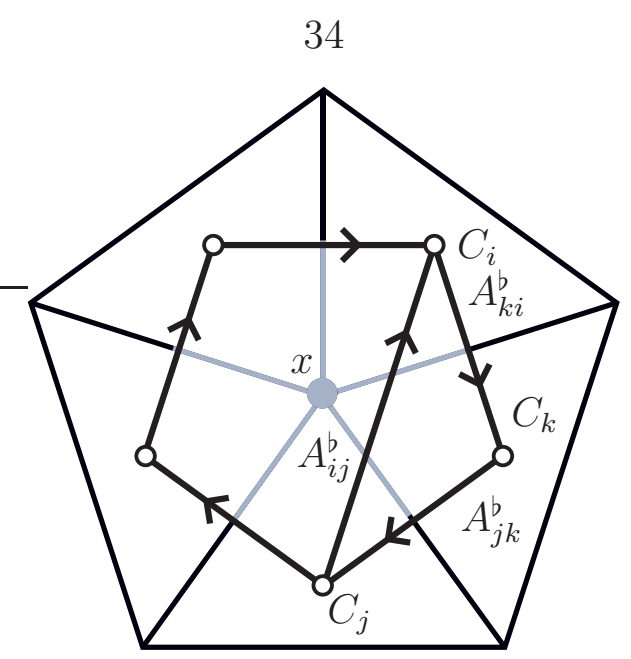

Figure 2.2: Flat Operator: schematic representation of $A_{i j}^{b}$ as a part of the cell dual to node $x$.

$C_{j}$. Thus, $\left(u\left(x_{i j}\right), \vec{n}_{i j}\right) S_{i j}$ is an approximation to $* u^{b}$ and the expression

$$
\frac{1}{2} \sum_{j}\left[\left(v\left(x_{i j}\right), \vec{n}_{i j}\right) h_{i j}\right]\left[\left(u\left(x_{i j}\right), \vec{n}_{i j}\right) S_{i j}\right]
$$

is a discrete version of $v^{b} \wedge * u^{b}$ in the sense of DEC [23, 33]. Therefore, the right side of the expression (2.48) is an approximation to the integral $\int_{M} v^{b} \wedge * u^{b}=\int_{M}(v, u)$ and condition (2.43) is satisfied.

Now, we need to define the terms $A_{i j}^{b}$ when cells $C_{i}$ and $C_{j}$ are "two cells away", i.e., when $j \notin N(i)$ and $N(i) \cap N(j) \neq \emptyset$. According to Lemma 5 , this has to be done in such a way that $\mathbf{d} A^{b}$ approximates the vorticity of the vector field.

We will use the fact that in the two-dimensional case

$$
\mathbf{d} v^{b}(u, w)=(* \omega)^{\sharp}\left(u^{b} \wedge w^{b}\right)^{\sharp} .
$$

Since for any matrix $X \in \mathcal{S}$, values of $X^{b}$ on neighboring cells can be considered as a dual 1-form in the sense of DEC, we can use DEC to construct approximations to $\mathbf{d} v^{b}$ and $u^{b} \wedge v^{b}$.

First, let's construct an approximation of vorticity. Suppose $j \notin N(i), k \in N(i) \cap$ $N(j)$ and cells $C_{i}, C_{j}$ and $C_{k}$ have a common vertex $x$. On the node $x$ we can define 


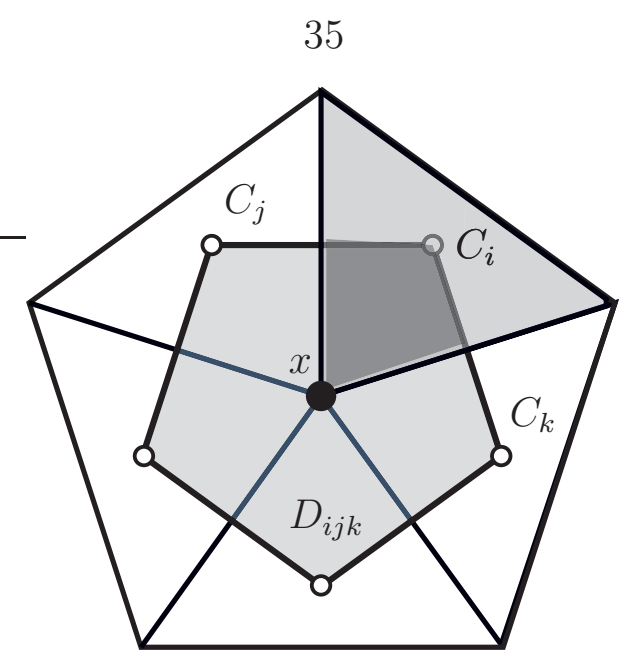

Figure 2.3: Ratio of Vorticity: schematic description of $K_{i j k}$ as the area ratio of $D_{i j k} \cap C_{i}$ and $D_{i j k}$.

a discrete vorticity in the sense of DEC:

$$
\omega_{\mathrm{DEC}}(x)=\frac{1}{\operatorname{vol}\left(D_{x}\right)}\left(A_{i_{1} i_{2}}^{b}+\ldots+A_{i_{p-1} i_{p}}^{b}\right) \approx \frac{1}{\operatorname{vol}\left(D_{x}\right)} \int_{D_{x}} d v^{b}
$$

where $i_{1}, \ldots, i_{p}$ is the collection of cells going counter-clockwise all around node $x$ and $D_{x}$ is the dual (Voronoi) cell centered at $x$.

We will define $A_{i j}^{b}$ to satisfy the equation

$$
\left(\mathbf{d} A^{\mathrm{b}}\right)_{i j k}=A_{i j}^{\mathrm{b}}+A_{j k}^{\mathrm{b}}+A_{k i}^{\mathrm{b}}=K_{i j k} \omega_{\mathrm{DEC}}(x) \operatorname{vol}\left(D_{x}\right),
$$

where $K_{i j k}$ is defined to be

$$
K_{i j k}=s_{i j k} \frac{\operatorname{vol}\left(D_{i j k} \cap C_{i}\right)}{\operatorname{vol}\left(D_{i j k}\right)},
$$

where $D_{i j k}$ is the dual cell centered around the common node to the cells $C_{i}, C_{j}$ and $C_{k}$ and $s_{i j k}=1$ if the triplet of cells $i, j$ and $k$ is oriented counter-clockwise and $s_{i j k}=-1$ if it's oriented clockwise.

When $h$ is small, $\omega_{\mathrm{DEC}}(x)$ is close to $\omega\left(x_{i}\right)$, where $x_{i}$ is the barycenter of cell $i$. Thus, given our definition of the discrete flat operator, the expression for every cell 
$C_{i}$

$$
\sum_{j, k}\left(\mathbf{d} A^{b}\right)_{i j k} B_{i k} C_{i j}
$$

is close to

$$
\omega\left(x_{i}\right) \sum_{j, k} K_{i j k} \operatorname{vol}\left(D_{i j k}\right) B_{i k} C_{i j}=\omega\left(x_{i}\right) \frac{1}{\Omega_{i}} \sum_{j, k} s_{i j k} \frac{\operatorname{vol}\left(D_{i j k} \cap C_{i}\right)}{\Omega_{i}}\left(\frac{S_{i k}}{h_{i k}} B_{i k}^{b}\right)\left(\frac{S_{i j}}{h_{i j}} C_{i j}^{b}\right) .
$$

Now, since for a matrix $X \in \mathcal{S}$ the values $X_{i j}^{b}$ on neighboring cells $i$ and $j$ can be considered as a dual one-form in the sense of DEC (i.e., values $X_{i j}^{b}$ are associated to the dual edges), the values $\frac{S_{i j}}{h_{i j}} X_{i j}^{b}$ can be considered as a primal 1 -form $* X^{b}$. Also, the expression

$$
\frac{1}{2} \sum_{j, k} s_{i j k} \frac{\operatorname{vol}\left(D_{i j k} \cap C_{i}\right)}{\Omega_{i}} X_{i k}^{b} Y_{i j}^{b}
$$

is a version of primal-primal wedge product, i.e., if $X^{b}$ and $Y^{b}$ are primal one-forms, then

$$
\frac{1}{2} \sum_{j, k} s_{i j k} \frac{\operatorname{vol}\left(D_{i j k} \cap C_{i}\right)}{\Omega_{i}} X_{i k}^{b} Y_{i j}^{b}=\left(X^{b} \wedge Y^{b}\right)_{i}
$$

This definition of wedge product was used, for instance, in [33, 23].

Therefore, we can see that

$$
\begin{aligned}
& \left\langle\left\langle\mathbf{d} A^{b},(B, C)\right\rangle=\frac{1}{2} \sum_{i, j, k} \Omega_{i}\left(\mathbf{d} A^{b}\right)_{i j k} B_{i k} C_{i j} \approx\right. \\
& \frac{1}{2} \sum_{i} \omega\left(x_{i}\right) \sum_{j, k} s_{i j k} \frac{\operatorname{vol}\left(D_{i j k} \cap C_{i}\right)}{\Omega_{i}}\left(\frac{S_{i k}}{h_{i k}} B_{i k}^{b}\right)\left(\frac{S_{i j}}{h_{i j}} C_{i j}^{b}\right)=\sum_{i} \omega\left(x_{i}\right)\left(* B^{b} \wedge * C^{b}\right)_{i} .
\end{aligned}
$$

Therefore, since $* u \wedge * v=u \wedge w$, we get

$$
\sum_{i j, k} \Omega_{i}\left(d A^{b}\right)_{i j k} B_{i k} C_{i j} \approx \int(* \omega)^{\sharp} u^{b} \wedge w^{b}=\int_{M} \omega(u, w) .
$$

Note that any other convergent definition of the discrete wedge product would lead to a(nother) valid flat operator $b: A \mapsto A^{b}$. 


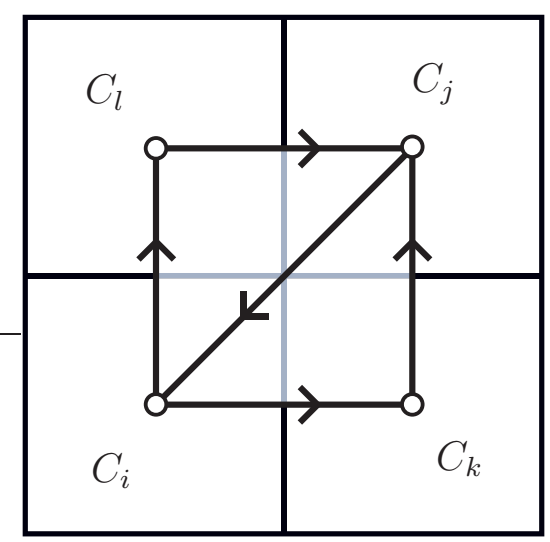

Figure 2.4: Flat Operator on a Regular Grid: our definition of the flat operator is particularly simple when the spatial discretization is a regular mesh.

Example: flat operator on a regular mesh. The following lemma introduces an explicit formula for a flat operator on a regular grid.

Lemma 6. For a domain represented with a Cartesian grid of size $h$, let $A$ be an antisymmetric doubly-null matrix satisfying the NHC. The operator $b_{h}: A \mapsto A^{b_{h}}$ defined as

$$
\begin{gathered}
A_{i j}^{b}=h^{2} A_{i j}, \quad \text { for } i \in N(j), \\
A_{i j}^{b}=\frac{h^{2}}{\sharp(N(i) \cap N(j))} \sum_{k \in N(i) \cap N(j)}\left(A_{i k}+A_{k j}\right), \quad \text { for } i \neq N(j)
\end{gathered}
$$

is a discrete flat operator.

We can see that while $A$ satisfies the NHC, $A^{b}$ has non-zero elements for neighboring cells and for cells having a common neighbor (i.e., two cells away). Now, let $i, k, j, l$ be four cells on a regular mesh sharing a common node $x$, oriented counterclockwise. Then it is easy to see that for $A^{\text {b }}$ defined above we have

$$
\mathbf{d} A_{i j k}^{b}=\frac{1}{2} h^{2}\left(A_{i k}+A_{k j}+A_{j l}+A_{l i}\right)=\frac{\omega_{d}(x)}{2}
$$

where $\omega_{d}(x)$ is the discrete vorticity in the sense of Discrete Exterior Calculus integrated over the dual cell of node $x$. Since $\omega_{d}$ converges to vorticity, the condition of Lemma 5 is satisfied. 
Construction of a flat operator on a 3-dimensional mesh. Now we will describe a construction of a flat operator on a 3-dimensional simplicial grid. In the two-dimensional case the formula $* u \wedge * v=u \wedge v$ allowed us to use a discrete primalprimal wedge product to construct $A^{b}$. This is no longer possible in higher dimensions, so now we will use a discrete dual-dual wedge product to construct $A^{b}$. The procedure described below can be generalized to higher dimensions.

Just like in the two-dimensional case, we need to find a matrix $A^{b}$ which satisfies the properties

$$
\left\langle\left\langle A^{b}, A\right\rangle\right\rangle \rightarrow \int_{M} v^{2} d x
$$

and

$$
\left\langle\left\langle d A^{b},(B, C)\right\rangle\right\rangle \rightarrow \int_{M} \omega(u, w) d V .
$$

To satisfy the first property we define, as above,

$$
A_{i j}^{b}=A_{i j} \Omega_{i} \frac{h_{i j}}{S_{i j}}, \quad \text { when } j \in N(i)
$$

To satisfy the second property we will use the fact that

$$
\int_{M} \omega(u, w) d V=\int_{M} * \omega \wedge u^{b} \wedge v^{b}
$$

Let $\mathrm{A}^{b}$ be defined by formula

$$
\left(\mathbf{d} A^{b}\right)_{i j k}=A_{i j}^{b}+A_{j k}^{b}+A_{k i}^{b}=K_{i j k} \omega_{\mathrm{DEC}}\left(e_{i j k}\right) \operatorname{vol}\left(D_{i j k}\right),
$$

where $e_{i j k}$ is the primal edge, common to the cells $i, j, k$, and $D_{i j k}$ is the twodimensional face dual to $e_{i j k}$. Then the pairing $\left\langle\left\langle d A^{b},(B, C)\right\rangle\right\rangle$ can be written as

$$
\left\langle\left\langle d A^{b},(B, C)\right\rangle\right\rangle=\frac{1}{2} \sum_{i, j, k} \Omega_{i} K_{i j k} \omega_{\mathrm{DEC}}\left(e_{i j k}\right) \operatorname{vol}\left(D_{i j k}\right) B_{i k} C_{i k},
$$

or

$$
\left\langle\left\langle d A^{b},(B, C)\right\rangle\right\rangle=\frac{1}{2} \sum_{i, j, k} \Omega_{i} \tilde{K}_{i j k} \omega_{\mathrm{DEC}}\left(e_{i j k}\right) \operatorname{vol}\left(D_{i j k}\right) B_{i k}^{b} C_{i j}^{b},
$$


where

$$
B_{i j}^{b}=\Omega_{i} B_{i j} \frac{h_{i} j}{S_{i j}}, \quad C_{i j}^{b}=\Omega_{i} C_{i j} \frac{h_{i j}}{S_{i j}}
$$

and

$$
\tilde{K}_{i j k}=K_{i j k} \frac{1}{\Omega_{i}^{2}} \frac{S_{i j}}{h_{i j}} \frac{S_{i k}}{h_{i j}}
$$

Now, suppose we have a discrete version (in the sense of DEC) of the wedge product between two dual one-forms, written as

$$
\left(B^{b} \wedge C^{b}\right)_{D_{e}}=\sum T_{i j k} B_{i k}^{b} C_{i j}^{b}
$$

where $D_{e}$ is a dual two-face intersecting a primal edge $e$ and the sum is taken over all cells $i, j$ and $k$ which have $e$ as a common edge. Then the equality (2.51) can be written as

$$
\left\langle\left\langle d A^{b},(B, C)\right\rangle\right\rangle=\frac{1}{2} \sum_{e} \omega_{\mathrm{DEC}}(e)|e| \sum_{\substack{i, j, k \\ e=C_{i} \cap C_{j} \cap C_{k}}}\left(B^{b} \wedge C^{b}\right)_{D_{e}} T_{i j k}^{-1} \tilde{K}_{i j k} \Omega_{i} \operatorname{vol}\left(D_{e}\right)|e|^{-1} .
$$

Here $|\cdot|$ is the $k$-dimensional volume of a $k$-cell. So, if we define

$$
\tilde{K}_{i j k}=T_{i j k} \Omega_{i}^{-1} \operatorname{vol}\left(D_{e}\right)^{-1}|e|,
$$

we have

$$
\left\langle\left\langle d A^{b},(B, C)\right\rangle\right\rangle=\frac{1}{2} \sum_{e} \omega_{\mathrm{DEC}}(e)|e|\left(B^{b} \wedge C^{b}\right)_{D_{e}} \approx \int_{M}(* \omega) \wedge u^{b} \wedge w^{b} .
$$

Thus, for any set of coefficients $T_{i j k}$ we get a formula to compute $A^{\text {b }}$ :

$$
\left.A_{i j}^{b}+A_{j k}^{b}+A_{k i}^{b}=K_{i j k} \omega_{\mathrm{DEC}}\left(e_{i j k}\right)\right) \operatorname{vol}\left(D_{i j k}\right),
$$

where

$$
K_{i j k}=T_{i j k} \Omega_{i} \frac{h_{i j} h_{i k}}{S_{i j} S_{i k}} \frac{|e|}{\operatorname{vol}\left(D_{e}\right)} .
$$


We can obtain a particular choice of $T_{i j k}$ by extending in an obvious way the definition of primal-primal wedge product given in [23]:

$$
T_{i j k}=s_{i j k} \frac{1}{2} \frac{\left|D_{i j k} \cap C_{i}\right|}{\left|\Delta_{i j k}\right|}
$$

where $\Delta_{i j k}$ is a triangle with vertices at the centers of the cells $C_{i}, C_{j}$ and $C_{k}$.

To simplify the expression for $K_{i j k}$ we use the equality $\left|\Delta_{i j k}\right|=\frac{1}{2} h_{i j} h_{i k} \sin \alpha_{i j k}$, where $\alpha_{i j k}$ is the angle between dual edges:

$$
K_{i j k}=s_{i j k} \Omega_{i}|e| \frac{1}{\sin \alpha_{i j k}} \frac{S_{i j}}{S_{i k}} \frac{\left|D_{i j k} \cap C_{i}\right|}{\left|D_{j}\right|} .
$$

Now, using the generalized law of cosines for the volume of a tetrahedron yields

$$
\Omega_{i}=\frac{2}{3} \frac{1}{|e|} S_{i j} S_{i k} \sin \alpha_{i j k}
$$

and thus

$$
K_{i j k}=\frac{2}{3} s_{i j k} \frac{\left|D_{i j k} \cap C_{i}\right|}{\left|D_{i j k}\right|} .
$$

This formula was used in the implementation of our method described in [30].

\subsection{Dynamics on the Group of $\Omega$-Orthogonal Sto- chastic Matrices}

We now focus on defining a Lagrangian on the tangent bundle of the group $\mathcal{D}(\mathbb{M})$

of $\Omega$-orthogonal, signed stochastic matrices and studying the corresponding variational principle with non-holonomic constraints. We will first assume a discretespace/continuous-time setup before presenting a fully discrete version.

\subsubsection{Variational principle}

We wish to study dynamics on the Lie group $\mathcal{D}(\mathbb{M})$ of $\Omega$-orthogonal, signed stochastic matrices representing volume-preserving diffeomorphisms on a mesh $\mathbb{M}$. While 
the group's Lie algebra $\mathfrak{D}(\mathbb{M})$ consists of column-null $\Omega$-antisymmetric matrices, we restrict the Eulerian velocity $A=\dot{q} q^{-1}$ to lie in the NHC space $\mathcal{S}$, i.e., with fluid transfer only between adjacent cells (see Section 2.1.4).

We first define a Lagrangian $L_{h}(q, \dot{q})$ on $T \mathcal{D}(\mathbb{M})$ such that $L_{h} \underset{h \rightarrow 0}{\longrightarrow} \frac{1}{2} \int\|v\|^{2}$ for $A \underset{h \rightarrow 0}{\rightsquigarrow} v:$

$$
L_{h}(A)=\frac{1}{2}\left\langle\left\langle A^{b}, A\right\rangle \equiv \frac{1}{4} \operatorname{Tr}\left(\Omega A^{b} A^{T}\right) .\right.
$$

When $A$ satisfies the NHC, it was shown in Section 2.2.5 that $\left\langle\left\langle A^{b}, A\right\rangle\right\rangle \rightarrow \int(v, v)$; thus the discrete Lagrangian is an approximation to the $L_{2}$-norm of the velocity field in this case.

To compute the variation of $A(t)$, we assume that $q$ depends on a parameter $s$, we denote $q^{\prime}=\frac{d q}{d s}$ and $\dot{q}=\frac{d q}{d t}$, and we differentiate the Eulerian velocity:

$$
\frac{d}{d s} A(s, t)=\dot{q}^{\prime} q^{-1}-\dot{q} q^{-1} q^{\prime} q^{-1} .
$$

Now, if we denote by $B$ the vector field satisfying $B=q^{\prime} q^{-1}$, we get the well-known Lin constraints:

$$
\frac{d}{d s} A(s, t)=\dot{B}-[A, B]
$$

where $[A, B]=A B-B A$ is the commutator of matrices.

Now remember that the dynamics of systems with non-holonomic constraints can be derived from the Lagrange-d'Alembert principle:

$$
\delta \int_{0}^{1} L_{h}(q, \dot{q}) d t=0 \text { with }\left\{\begin{array}{l}
\delta q \in S_{q} \\
\delta q(0)=\delta q(1)=0 .
\end{array}\right.
$$

Since $\delta q \in S_{q}$, the vector field $B$ must be in $\mathcal{S}$, i.e., $B_{i j}=0$ except for neighboring 
$C_{i}$ and $C_{j}$. We can then compute $\delta L_{h}$ :

$$
\delta L_{h}(A)=\frac{1}{2}\left(\left\langle\left\langle\delta A^{b}, A\right\rangle\right\rangle+\left\langle\left\langle A^{b}, \delta A\right\rangle\right\rangle\right)
$$

As we restrict $A$ to lie in the NHC subspace $\mathcal{S}$, the Lin constraints in Eq. (2.53) imply $\left\langle\left\langle\delta A^{b}, A\right\rangle\right\rangle=\left\langle\left\langle\dot{B}^{b}-[A, B], A\right\rangle\right\rangle=\left\langle\left\langle\dot{B}^{b}, A\right\rangle\right\rangle=\left\langle\left\langle A^{b}, \dot{B}\right\rangle\right\rangle$, and we get

$$
-2 \delta L_{h}(A)=-\operatorname{Tr}\left(\Omega A^{b} \dot{B}^{T}\right)+\frac{1}{2} \operatorname{Tr}\left(\Omega A^{b}[A, B]^{T}\right)=\operatorname{Tr}\left(A^{b} \Omega \dot{B}\right)-\frac{1}{2} \operatorname{Tr}\left(A^{b} \Omega[A, B]\right),
$$

which can be written as

$$
\delta L_{h}(A)=\left\langle\left\langle A^{b}, \dot{B}-\frac{1}{2}[A, B]\right\rangle\right\rangle
$$

Now, if $A$ is close to $-L_{v}$ and $B$ is close to $-L_{\xi}$ then, by definition of $b$, we have

$$
\left\langle\left\langle A^{b}, \dot{B}\right\rangle\right\rangle \rightarrow \int_{M}(v, \dot{\xi})
$$

and

$$
\left\langle\left\langle A^{b},[A, B]\right\rangle\right\rangle \rightarrow 2 \int_{M}(v,[\xi, v]) .
$$

Thus,

$$
\delta L_{h}(A) \rightarrow \int_{M}(v, \dot{\xi}+[v, \xi])=\delta \mathcal{L}
$$

so the discrete Lagrangian (resp., its variation) is an approximation of the continuous Lagrangian (resp., its variation).

Remark We know that the commutator $[A, B]$ is an approximation to the commutator of vector fields $[\xi, v]$. But after taking the variation of $L_{h}$ we obtain $\left\langle\left\langle A^{b}, \dot{B}-\right.\right.$ $\left.\left.\frac{1}{2}[A, B]\right\rangle\right\rangle$ and in order to obtain a correct approximation of Euler equation this variation has to approximate $\delta \mathcal{L}=\int_{M}(v, \dot{\xi}+[v, \xi])$. The pairing $\left\langle\left\langle A^{b},[A, B]\right\rangle\right\rangle$ thus approximates $2 \int_{M}(v,[\xi, v])$. 
Since for any matrices $A, B, C, \operatorname{Tr}(A[B, C])=\operatorname{Tr}([A, B] C)$, we get

$$
\delta L_{h}(A)=-\frac{1}{2} \operatorname{Tr}\left(A^{\mathrm{b}} \Omega \dot{B}-\frac{1}{2}\left[A^{\mathrm{b}} \Omega, A\right] B\right) .
$$

Therefore, after integration by parts, the discrete Euler-Lagrange equations of Eq. (2.29) are:

$$
\forall B \in \mathcal{S}, \quad \operatorname{Tr}\left(\left(2 \dot{A}^{b} \Omega+\left[A^{b} \Omega, A\right]\right) B\right)=0 .
$$

In order to express the resulting equations in a more intuitive fashion, we introduce the following lemma:

Lemma 7. If $A$ is doubly-null and antisymmetric, with $\operatorname{Tr}\left(B^{T} A\right)=0$ for every $B \in \mathcal{S}$ then there exists a discrete pressure field, i.e., a vector $P=\left(p_{1}, \ldots, p_{N}\right)$ such that

$$
A_{i j}=p_{i}-p_{j}, \quad \text { where } j \in N(i) .
$$

Elements $A_{i j}$ for non-neighboring cells $C_{i}$ and $C_{j}$ can be arbitrary.

Proof. Since $B \in \mathcal{S}$, the inner product of matrices $\operatorname{Tr}\left(B^{T} A\right)$ does not depend on $A_{i j}$ when $i$ and $j$ are not direct neighbors. Now, let's assume that $A \in \mathcal{S}$. The space $\mathcal{S}$ has codimension $N$ in the space $\mathcal{N}$. Indeed, it is defined by a system of $N$ independent equations:

$$
\sum_{j \in N(i)} B_{i j}=0, \quad 1 \leq i \leq N .
$$

Moreover, the space of discrete gradients $A_{i j}=p_{i}-p_{j}$ in $\mathcal{N}$ is orthogonal to the space of column-null antisymmetric matrices w.r.t. the inner product $A \cdot B=\operatorname{Tr}\left(B^{T} A\right)$ and has dimension $N$. Therefore, the orthogonal complement to $\mathcal{S}$ in $\mathcal{N}$ coincides with the space of discrete gradients.

Therefore, we deduce our main theorem: 
Theorem 1. Consider the discrete-space/continuous time Lagrangian on $T \mathcal{D}(\mathbb{M})$ :

$$
L_{h}(A)=\frac{1}{2}\left\langle\left\langle A^{b}, A\right\rangle\right\rangle
$$

where $A=\dot{q} q^{-1} \in \mathcal{S}$ is a column-null $\Omega$-antisymmetric matrix, $q \in \mathcal{D}(\mathbb{M})$ is a signed stochastic $\Omega$-orthognal matrix, and $A^{b}$ is the discrete flat operator defined in Def. 7 applied to $A$. Then the Lagrange-d'Alembert principle

$$
\delta \int_{0}^{1} L_{h}(A) d t=0, \quad \delta q \in S_{q}, \quad A \in S
$$

implies

$$
\left(\dot{A}^{b}+L_{A} A^{b}+\mathbf{d} \bar{p}\right)_{i j}=0, \quad \text { for } j \in N(i),
$$

or, equivalently,

$$
\dot{A}_{i j}+\frac{1}{2} \frac{S_{i j}}{\Omega_{i} \Omega_{j} h_{i j}}\left[A^{b} \Omega, A\right]_{i j}+\frac{S_{i j}}{\Omega_{i} h_{i j}}\left(p_{i}-p_{j}\right)=0, \quad \text { for } j \in N(i)
$$

where $p$ is the discrete pressure field and $\bar{p}$ is the modified discrete pressure, related to $p$ by the following formula (compare with Eq. (1.12))

$$
\bar{p}_{i}=p_{i}-\frac{1}{2}\left(A^{b} A\right)_{i i}
$$

Proof. Apply Lemma 7 (for $\Omega A$ and $B$ ) to Eq. (2.56) and substitute the definition of discrete Lie derivative given in Eq. (2.41).

We can see that these two forms of the resulting equations of motion clearly mimic the Euler equations, expressed respectively as

$$
\dot{v}^{b}+L_{v} v^{b}+\mathbf{d} \bar{p}=0
$$

and

$$
\dot{v}+\nabla_{v} v+\nabla p=0
$$




\subsubsection{Discrete Kelvin's theorem}

In this section we present a discrete version of Kelvin's theorem, by replacing the notions of a curve and its advection by their discrete Eulerian counterparts. After we introduce notions of discrete curve and discrete advection, the proof of Kelvin's theorem will be essentially the same as in the continuous case, which we will describe first for completeness.

\subsubsection{Continuous property}

Kelvin's theorem states that the circulation along a closed curve stays constant as the curve is advected with the flow. Let $\gamma_{t}$ be the curve and $C_{\gamma_{t}} v_{t}$ be the circulation of $v_{t}$ along $\gamma_{t}$ :

$$
C_{\gamma_{t}} v_{t}=\oint_{\gamma_{t}} v_{t} \cdot d s
$$

Let's consider a divergence-free vector field $\gamma_{0}^{\varepsilon}$ representing a "narrow current" of size $\varepsilon$ flowing along $\gamma_{0}$ and which has flux $\varepsilon$ in the direction transverse to the curve. And let $\gamma_{t}^{\varepsilon}$ be the field $\gamma_{0}^{\varepsilon}$ advected by the flow $v_{t}$, i.e., it satisfies equation

$$
\dot{\gamma}_{t}^{\varepsilon}+L_{v_{t}} \gamma_{t}^{\varepsilon}=0
$$

Then, as $\varepsilon \rightarrow 0$,

$$
\left\langle v_{t}^{b}, \gamma_{t}^{\varepsilon}\right\rangle \rightarrow C_{\gamma_{t}} v_{t}
$$

so the pairing $\left\langle v_{t}^{b}, \gamma_{t}^{\varepsilon}\right\rangle$ can be considered as an approximate circulation. We can compute its derivative:

$$
\frac{d}{d t}\left\langle v_{t}^{b}, \gamma_{t}^{\varepsilon}\right\rangle=\left\langle\dot{v}_{t}^{b}, \gamma_{t}^{\varepsilon}\right\rangle+\left\langle v_{t}^{b}, \dot{\gamma}_{t}^{\varepsilon}\right\rangle=-\left\langle L_{v_{t}} v_{t}^{b}, \gamma_{t}^{\varepsilon}\right\rangle-\left\langle v_{t}^{b}, L_{v_{t}} \gamma_{t}^{\varepsilon}\right\rangle=0
$$

And since this pairing represents circulation, the circulation itself stays constant. 


\subsubsection{Discrete Kelvin's theorem}

We now show that our discrete Euler equations give rise to a discrete analog of Kelvin's circulation theorem. Achieving this goal first requires a discrete definition of curves and their advection, for which we will borrow the concept of one-chains used in algebraic topology and demonstrate that curves and vector fields satisfying the non-holonomic constraints share the same representation; that is, the discretization of a curve $\gamma(s)$ will be thought of as a discretization of the narrow current $\gamma^{e}$. Since we already have established a discrete analog to Lie derivative (the commutator of matrices), we will be able to define how to advect a discrete curve along a discrete vector field. We will find that, just like Kelvin's circulation theorem in the continuous case, for any discrete curve $\gamma_{t}$ advected by a discrete vector $A(t)$ field satisfying the discrete Euler equation, the circulation of $A(t)$ along $\gamma_{t}$ is constant.

Discrete curves. A discrete curve in our Eulerian setup can be nicely defined using the concept of one-chains. Let's recall that a dual one-chain (see [31] for a thorough exposition of chains and simplicial homology) is a linear combination of the dual edges between cells. In our context, we will represent this linear combination as an $\Omega$-antisymmetric matrix. We start by defining a simple curve:

Definition 8. A simple discrete curve consisting of a discrete path from cell $C_{i_{1}}$ to cell $C_{i_{2}}, \ldots$, to cell $C_{i_{n}}$ is represented by a $\Omega$-antisymmetric matrix $B$ such that

$$
\Omega_{i_{k}} B_{i_{k} i_{k+1}}=-B_{i_{k+1} i_{k}} \Omega_{i_{k+1}}=\frac{1}{2}
$$

and

$$
B_{i j}=0, \quad \text { for }(i, j) \neq\left(i_{k}, i_{k+1}\right) \forall k \text {. }
$$

The matrix $B$ representing a simple discrete curve $\gamma(s)$ (that exactly follows dual edges) can be considered as a discrete version of the velocity $d \gamma(s) / d s$. Furthermore, one can extend the notion of discrete curves to encompass arbitrary dual one-chains (see Bossavit's description in, for instance, [7]). In our work, we will be focusing on closed discrete curves described as linear combinations of dual edges: 
Definition 9. A discrete closed curve is represented by a column-null $\Omega$-antisymmetric matrix $B$ such that $B_{i j}=0 \forall i \neq N(j)$.

Notice that this representation of a one-chain coincides with our definition of discrete Eulerian velocities in the NHC. We will therefore no longer distinguish between discrete curves and discrete velocities as they are represented by the same discrete object.

Discrete circulation. To compute circulation of a vector field along a curve, we apply the same pairing of matrices we used earlier:

Definition 10. The circulation $C_{B} A$ of a discrete vector field $A$ along a discrete curve $B$ is defined as

$$
C_{B} A=\left\langle\left\langle A^{b}, B\right\rangle\right\rangle
$$

Now, we need to define a discrete notion of advection, which should be an approximation to $L_{v} \gamma^{\varepsilon}$. We use a matrix $A \in \mathcal{S}$ to discretize $v$ and a matrix $B \in \mathcal{S}$ to discretize $\gamma^{\varepsilon}$, so their commutator $[A, B]$ is a discretization of $L_{v} \gamma^{\varepsilon}$. However, $[A, B] \notin \mathcal{S}$. So instead, we construct a matrix from elements of $[A, B]$ that satisfies the constraints and can still be considered as an approximation to the commutator of vector fields.

Definition 11. Let $B_{t} \in \mathcal{S}$ be a family of discrete curves evolving in time and $A_{t}$ is a (time-dependent) discrete vector field. We say that $B_{t} \in \mathcal{S}$ is advected by $A_{t}$ if $B_{t}$ satisfies the advection equation

$$
\left\langle\left\langle X^{b}, \dot{B}-\frac{1}{2}[A, B]\right\rangle\right\rangle=0, \quad \text { for any } X \in \mathcal{S} \text {. }
$$

First, let's see that the last equation has a solution. Indeed, $[A, B]_{i j}=0$ when $j \in N(i)$ and if the cells $C_{i}$ and $C_{j}$ are two-away (i.e., $\left.N(i) \cap N(j) \neq \emptyset\right), X_{i j}^{b}$ can be written as a linear combination:

$$
X_{i j}^{b}=\sum_{k} \alpha_{k}^{i j} X_{m_{k}^{i j} n_{k}^{i j}}^{b}
$$




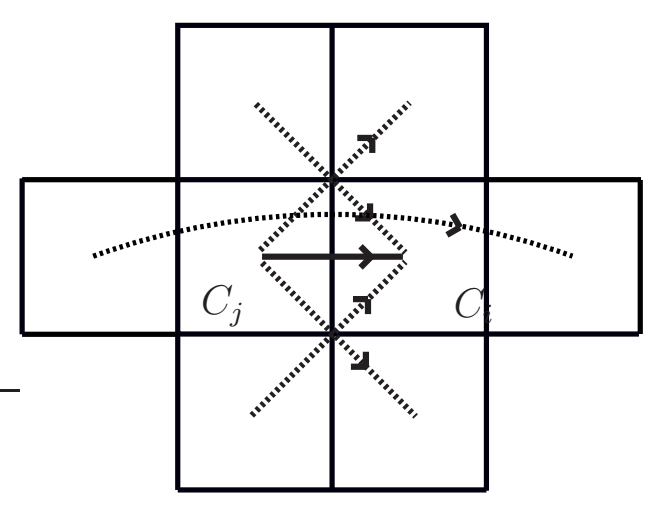

Figure 2.5: Projection on Regular Grids: our projection of $[A, B]$ onto the subspace of non-holonomic constraints accumulates on the common boundary of $C_{i}$ and $C_{j}$ all the two-cell-away transfers going through this boundary.

where $m_{k}^{i j} \in N\left(n_{k}^{i j}\right) \forall k$. Since $\dot{B} \in \mathcal{S}$, Eq. (2.61) can be written as

$$
\sum_{\substack{p, q \\ p \in N(q)}} X_{p q}^{b} \dot{B}_{p q} \Omega_{p}-\frac{1}{2} \sum_{\substack{i, j \\ N(i) \cap N(j) \neq \emptyset}} \Omega_{i}[A, B]_{i j} \sum_{k} \alpha_{k}^{i j} X_{m_{k}^{i j} n_{k}^{i j}}^{b}=0 .
$$

By grouping terms containing $X_{p q}^{b}$ we get

$$
\sum_{\substack{p . q \\ q \in N(p)}} X_{p q}^{b}\left(\dot{B}_{p q} \Omega_{p}-\sum_{r^{p q}, s^{p q}} \Omega_{r^{p q}}[A, B]_{r^{p q} s^{p q}} \alpha_{r^{p q}} s^{p q}\right)=0
$$

which has a solution

$$
\dot{B}_{p q}=\Omega_{p}^{-1} \sum_{r^{p q}, s^{p q}} \Omega_{r^{p q}}[A, B]_{r^{p q} s^{p q}} \beta_{r^{p q} s^{p q}}
$$

Now, let's prove that if $\dot{B}$ satisfies Eq. (2.61), it is an approximation to $L_{v} \gamma$. Indeed, if $X \rightsquigarrow w, A \rightsquigarrow v, B \rightsquigarrow \gamma$, then, by definition of the discrete operator $b$,

$$
\left\langle\left\langle X^{b}, \dot{B}\right\rangle\right\rangle \rightarrow \int_{M}(w, \gamma)
$$

and

$$
\left\langle\left\langle X^{b},[A, B]\right\rangle\right\rangle \rightarrow 2 \int_{M}(x,[u, \gamma])
$$


Thus, if Eq. (2.61) is satisfied, $\dot{\gamma}$ has to satisfy

$$
\int_{M}(w, \dot{\gamma}-[u, \gamma])=0
$$

for every $w \in \operatorname{SVect}(M)$. Since $\gamma \in \operatorname{SVect}(M)$, it means that $\dot{\gamma}=[u, \gamma]=L_{u} \gamma$.

Discrete Kelvin's theorem. We are now ready to give a discrete analog of Kelvin's circulation theorem satisfied by our discrete Euler equations.

Theorem 2. If $A_{t}$ satisfies the DEL equations (2.57) and $B_{0}$ is an arbitrary discrete curve, then the circulation of $A$ along $B_{t}$ stays constant:

$$
C_{B_{t}} A_{t}=C_{B_{0}} A_{0}
$$

where $B_{t}$ is the curve $B_{0}$ advected by $A_{t}$.

Proof. The time derivative of the circulation $C_{B_{t}} A_{t}$ is expressed as:

$$
\frac{d}{d t} C_{B_{t}} A_{t}=\frac{d}{d t}\left\langle\left\langle A_{t}^{b}, B_{t}\right\rangle\right\rangle=\left\langle\left\langle\dot{A}_{t}^{b}, B_{t}\right\rangle\right\rangle+\left\langle\left\langle A_{t}^{b}, \dot{B}_{t}\right\rangle\right\rangle
$$

Since $A_{t}^{b}$ satisfies the DEL equations $\left(\dot{A}_{t}^{b}+\frac{1}{2}\left[A_{t}^{b} \Omega, A_{t}\right] \Omega^{-1}+\mathbf{d} \bar{p}_{t}\right)_{i j}=0$ for $i$ and $j$ representing two neighboring cells' indices, and as $\operatorname{Tr}\left(\left(\mathbf{d} \bar{p}_{t}\right) B_{t}\right)=0$, we have

$$
\begin{aligned}
\left\langle\left\langle\dot{A}_{t}^{b}, B_{t}\right\rangle\right\rangle=-\frac{1}{2}\left\langle\left\langle\left[A_{t}^{b} \Omega, A_{t}\right] \Omega^{-1}, B_{t}\right\rangle\right\rangle=- & \frac{1}{2} \operatorname{Tr}\left(\left[A_{t}^{b} \Omega, A_{t}\right] B_{t}\right)= \\
& \quad-\frac{1}{2} \operatorname{Tr}\left(A_{t}^{b} \Omega\left[A_{t}, B_{t}\right]\right)=-\frac{1}{2}\left\langle\left\langle A_{t}^{b},\left[A_{t}, B_{t}\right]\right\rangle\right\rangle .
\end{aligned}
$$

But since $B_{t}$ is advected by $A$, we get

$$
\frac{d}{d t} C_{B_{t}} A_{t}=\left\langle\left\langle\dot{A}_{t}^{b}, B_{t}\right\rangle\right\rangle+\left\langle\left\langle A_{t}^{b}, \dot{B}_{t}\right\rangle\right\rangle=\left\langle\left\langle A_{t}^{b}, \dot{B}_{t}-\frac{1}{2}\left[A_{t}, B_{t}\right]\right\rangle\right\rangle=0
$$

Remark. In the continuous case, the Kelvin's circulation theorem can be derived from Noether's theorem using right-invariance of the metric on SDiff (particle rela- 
belling symmetry). In the discrete case, the Lagrangian is also right invariant, but the presence of the non-holonomic constraints prevents us from using Noether's theorem. Indeed, in general, in a system with non-holonomic constraints a momentum is no longer expected to be conserved. But we can still use the symmetry to obtain the momentum equation, i.e. compute the rate of change of the momentum in time. Doing so for our discrete fluid model we also get our discrete circulation theorem.

\subsection{Fluid Evolution in Discrete Time}

We now revisit our discrete version on the variational principle discussed above where time is now discrete instead of continuous. We assume that the fully discrete fluid motion is given as a discrete path $q_{1}, \ldots, q_{K}$ in the space of $\Omega$-orthogonal signed stochastic matrices, where the motion has been sampled at regular time $t_{k}=k \tau$.

Discrete velocity. Given a pair $q_{k}, q_{k+1}$ of consecutive configurations in time, we can compute a discrete time analog of Eulerian velocity using, e.g., one of the following classical formulas:

$$
\begin{array}{lll}
\text { - } q_{k+1}-q_{k}=\tau A_{k} q_{k}, & & \text { (explicit Euler), } \\
\text { - } q_{k+1}-q_{k}=\tau A_{k} q_{k+1}, & & \text { (implicit Euler), } \\
\text { - } q_{k+1}-q_{k}=\tau A_{k} \frac{q_{k}+q_{k+1}}{2}, & & \text { (midpoint rule). }
\end{array}
$$

Note that the midpoint rule preserves the Lie group structure of the configuration space. Note also that many other discretizations could be used, but we will restrict our explanations to these simple cases as they suffice to illustrate how our continuous time procedure can be adapted.

Discrete Lagrangian and action. We define the discrete-space/discrete-time Lagrangian $L_{d}\left(q_{k}, q_{k+1}\right)$ as

$$
L_{d}\left(q_{k}, q_{k+1}\right)=L\left(A_{k}\right)
$$

The discrete action $\mathcal{A}_{d}$ along a discrete path is then simply the sum of all pairwise 


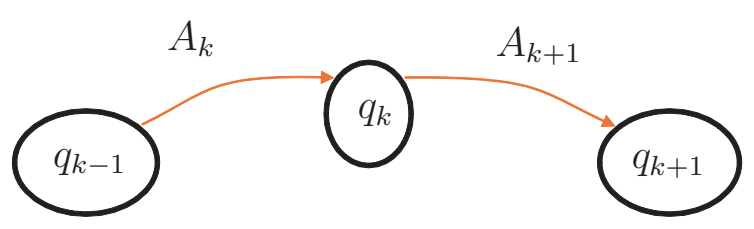

Figure 2.6: Three consecutive configurations $q_{k-1}, q_{k}, q_{k+1}$ of a fluid in time, with Eulerian velocities $A_{k}$ and $A_{k+1}$.

discrete Lagrangians:

$$
\mathcal{A}_{d}\left(q_{0}, \ldots, q_{K}\right)=\sum_{k=0}^{K-1} L_{d}\left(q_{k}, q_{k+1}\right)
$$

We can now use the Lagrange-d'Alembert principle that states that $\delta \mathcal{A}_{d}=0$ for all variations of the $q_{k}$ (for $k=1, \ldots, K-1$, with $q_{0}$ and $q_{K}$ being fixed) in $S_{q}$ while $A_{k}$ is restricted to $\mathcal{S}$.

Variations. The variations of this action in all three discrete advection cases become:

- Explicit Euler. In this case, $A_{k}=\left(q_{k+1}-q_{k}\right) / \tau q_{k}^{-1}$. The variation $\delta_{k} A_{k}$ and $\delta_{k+1} A_{k}$ with respect to $q_{k}$ and $q_{k+1}$ respectively become:

$$
\begin{gathered}
\delta_{k} A_{k}=-\frac{1}{\tau} \delta q_{k} q_{k}^{-1}-\frac{q_{k+1}-q_{k}}{\tau} q_{k}^{-1} \delta q_{k} q_{k}^{-1}, \\
\delta_{k+1} A_{k}=\frac{1}{\tau} \delta q_{k+1} q_{k}^{-1} .
\end{gathered}
$$

If we denote, similarly to the continuous case, $B_{k}=\delta q_{k} q_{k}^{-1}$, we get:

$$
\delta_{k} A_{k}=-\frac{B_{k}}{\tau}-A_{k} B_{k}
$$

and

$$
\delta_{k+1} A_{k}=\frac{B_{k+1}}{\tau}+B_{k+1} A_{k}
$$


- Implicit Euler. In this case $A_{k}=\left(q_{k+1}-q_{k}\right) / \tau q_{k+1}^{-1}$. It yields:

$$
\delta_{k} A_{k}=-\frac{1}{\tau} \delta q_{k} q_{k+1}^{-1}
$$

and

$$
\delta_{k+1} A_{k}=\frac{1}{\tau} \delta q_{k+1} q_{k+1}^{-1}-\frac{q_{k+1}-q_{k}}{\tau} q_{k+1}^{-1} \delta q_{k+1} q_{k+1}^{-1}
$$

Similarly to the previous case we now obtain:

$$
\delta_{k} A_{k}=-\frac{B_{k}}{\tau}+B_{k} A_{k}
$$

and

$$
\delta_{k+1} A_{k}=\frac{B_{k+1}}{\tau}-A_{k} B_{k+1}
$$

- Midpoint. The Eulerian velocity between $q_{k}$ and $q_{k+1}$ is now expressed as $A_{k}=$ $2 \frac{q_{k+1}-q_{k}}{\tau}\left(q_{k+1}+q_{k}\right)^{-1}$. Thus,

$$
\begin{aligned}
\delta_{k} A_{k}= & -2 \frac{\delta q_{k}}{\tau}\left(q_{k+1}+q_{k}\right)^{-1} \\
& -2 \frac{q_{k+1}-q_{k}}{\tau}\left(q_{k+1}+q_{k}\right)^{-1} \delta q_{k}\left(q_{k+1}+q_{k}\right)^{-1} \\
= & -\frac{1}{\tau}\left(2 B_{k}+\tau A_{k} B_{k}\right) q_{k}\left(q_{k+1}+q_{k}\right)^{-1} \\
= & -\frac{1}{\tau}\left(\mathrm{Id}+\frac{\tau}{2} A_{k}\right) B_{k}\left(\mathrm{Id}-\frac{\tau}{2} A_{k}\right) .
\end{aligned}
$$

Similarly, we get:

$$
\delta_{k+1} A_{k}=\frac{1}{\tau}\left(\mathrm{Id}-\frac{\tau}{2} A_{k}\right) B_{k+1}\left(\mathrm{Id}+\frac{\tau}{2} A_{k}\right)
$$

Discrete Euler-Lagrange equations. Equating the variations of the action $\mathcal{A}_{d}$ with respect to $\delta q_{k}$ to zero for $k \in[1, K-1]$ yields:

$$
\delta_{k}\left\langle\left\langle A_{k-1}^{b}, A_{k-1}\right\rangle\right\rangle+\delta_{k}\left\langle\left\langle A_{k}^{b}, A_{k}\right\rangle\right\rangle=0 .
$$


Similarly to the continuous time case, we have

$$
\left\langle\left\langle\delta_{k} A_{k}^{b}, A_{k}\right\rangle\right\rangle=\left\langle\left\langle A_{k}^{b},-B_{k} / \tau\right\rangle\right\rangle
$$

and

$$
\left\langle\left\langle\delta_{k} A_{k-1}^{b}, A_{k-1}\right\rangle\right\rangle=\left\langle\left\langle A_{k-1}^{b}, B_{k} / \tau\right\rangle\right\rangle
$$

Thus, Eq.(2.62) becomes

$$
\operatorname{Tr}\left[A_{k-1}^{b} \Omega\left(\delta_{k} A_{k-1}+B_{k} / \tau\right)+A_{k}^{b} \Omega\left(\delta_{k} A_{k}-B_{k} / \tau\right)\right]=0 .
$$

Now, let's solve it for $A_{k}$ in the explicit case. Substituting the expressions for $\delta_{k} A_{k}$ and $\delta_{k} A_{k-1}$ yields:

$$
\operatorname{Tr}\left[-A_{k}^{b} \Omega\left(2 B_{k}+\tau A_{k} B_{k}\right)+A_{k-1}^{b} \Omega\left(2 B_{k}+\tau B_{k} A_{k-1}\right)\right]=0 .
$$

Denoting $\dot{A}_{k}^{b}=\left(A_{k}^{b}-A_{k-1}^{b}\right) \tau^{-1}$ we can rewrite the last equation as

$$
\left.\operatorname{Tr}\left[\left(2 \dot{A}_{k}^{b} \Omega+A_{k}^{b} \Omega A_{k}-A_{k-1} A_{k-1}^{b} \Omega\right) B_{k}\right)\right]=0 .
$$

Therefore, we get the following discrete Euler-Lagrange equations in the explicit case:

$$
\dot{A}_{k}^{b}+\frac{1}{2}\left(\frac{A_{k}^{b} \Omega A_{k} \Omega^{-1}-A_{k-1} A_{k-1}^{b}}{2}-\frac{\left(A_{k}^{b} \Omega A_{k}-A_{k-1} A_{k-1}^{b}\right)^{T}}{2}\right)+\mathbf{d} p_{k}=0,
$$

which is equivalent to

$$
\dot{A}_{k}^{b}+\frac{1}{4}\left(\left[A_{k}^{b} \Omega, A_{k}\right] \Omega^{-1}+\left[A_{k-1}^{b} \Omega, A_{k-1}\right] \Omega^{-1}\right)+\mathbf{d} p_{k}=0 .
$$

Using the implicit formula for $A_{k}$ instead of the implicit one we get exactly the same equation, and we omit the computations here. 
The midpoint case is fairly similar. First, we get

$$
\left\langle\left\langle\delta_{k} A_{k}^{b}, A_{k}\right\rangle\right\rangle=\left\langle\left\langle A_{k}^{b},-B_{k} / \tau+\tau A_{k} B_{k} A_{k} / 4\right\rangle\right\rangle
$$

and

$$
\left\langle\left\langle\delta_{k} A_{k-1}^{b}, A_{k-1}\right\rangle\right\rangle=\left\langle\left\langle A_{k}^{b}, B_{k} / \tau-\tau A_{k-1} B_{k} A_{k-1} / 4\right\rangle\right\rangle .
$$

For the DEL equations we get

$$
\begin{aligned}
\operatorname{Tr}\left[A_{k}^{b} \Omega\left(-\frac{1}{\tau}\left(\mathrm{Id}+\frac{\tau}{2} A_{k}\right) B_{k}\left(\mathrm{Id}-\frac{\tau}{2} A_{k}\right)-B_{k} / \tau+\tau A_{k} B_{k} A_{k} / 4\right)+\right. \\
\left.A_{k-1}^{b} \Omega\left(\frac{1}{\tau}\left(\mathrm{Id}-\frac{\tau}{2} A_{k-1}\right) B_{k}\left(\mathrm{Id}+\frac{\tau}{2} A_{k-1}\right)+B_{k} / \tau-\tau A_{k-1} B_{k} A_{k-1} / 4\right)\right]=0
\end{aligned}
$$

which is the same as

$$
\begin{aligned}
\operatorname{Tr}\left[\left(\dot{A}_{k}^{b}+\frac{1}{4}\left(\left[A_{k}^{b} \Omega, A_{k}\right] \Omega^{-1}+\right.\right.\right. & {\left.\left[A_{k-1}^{b} \Omega, A_{k-1}\right] \Omega^{-1}\right)+ } \\
& \left.\left.\frac{\tau}{4}\left(A_{k} A_{k}^{b} \Omega A_{k} \Omega^{-1}-A_{k-1} A_{k-1}^{b} \Omega A_{k-1} \Omega^{-1}\right)\right) \Omega B_{k}\right]=0,
\end{aligned}
$$

therefore, the DEL equations become

$$
\begin{aligned}
\dot{A}_{k}^{b}+\frac{1}{4}\left(\left[A_{k}^{b} \Omega, A_{k}\right] \Omega^{-1}+\left[A_{k-1}^{b} \Omega, A_{k-1}\right] \Omega^{-1}\right)+ & \\
\frac{\tau}{4}\left(A_{k} A_{k}^{b} \Omega A_{k}^{T} \Omega^{-1}-A_{k-1} A_{k-1}^{b} \Omega A_{k-1}^{T} \Omega^{-1}\right)+\mathbf{d} p_{k} & =0 .
\end{aligned}
$$

This equation has terms $\left[A_{k}^{b}, A_{k}\right]+\left[A_{k-1}^{b}, A_{k-1}\right]$ converging to the Euler equation's term $\nabla_{v} v$, which are the same as in the explicit and implicit cases. It only differs from the other cases by the cubic term, which, in the limit of infinitesimal time steps $(\tau \rightarrow 0)$, converges to zero.

Update rule for regular grids. The discrete Euler equation we derived above turns out to be particularly simple when applied to a regular grid. Indeed, let's consider a regular (rectangular) grid of size $h$, on a two-dimensional domain for simplicity. 


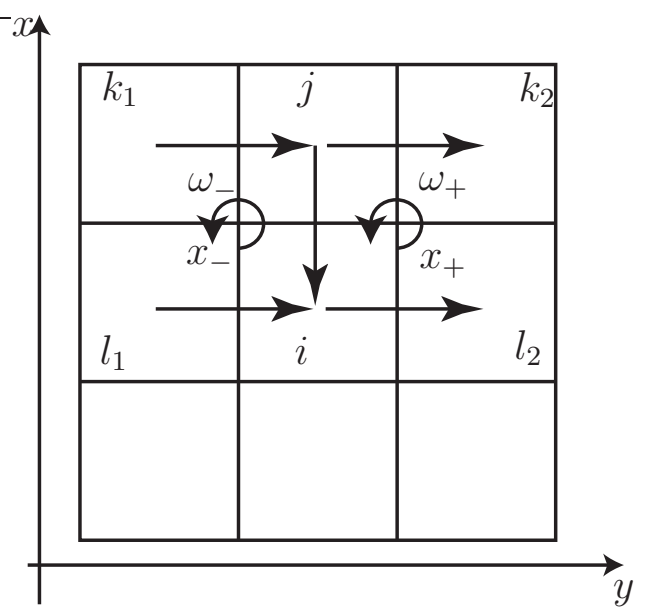

Figure 2.7: Discrete Euler equation on a regular mesh

Then the discrete Euler equation (2.58) becomes

$$
\dot{A}_{i j}+\frac{1}{2 h^{2}}\left[A^{b}, A\right]_{i j}+\frac{1}{h^{2}}\left(p_{i}-p_{j}\right)=0, \quad \text { where } j \in N(i) .
$$

Now let's fix $i$ and $j$ and compute $\left[A^{b}, A\right]_{i j}$. Since $A \in \mathcal{S}$ we have

$$
\left[A^{b}, A\right]_{i j}=\sum_{k \in N(j)} A_{i k}^{b} A_{k j}-\sum_{l \in N(i)} A_{i l} A_{l j}^{b} .
$$

From the definition on $A^{b}$ we get (see Fig. 2.4):

$$
A_{i k}^{b}=-\frac{1}{2} \omega_{i k} s_{i j k}+h^{2}\left(A_{i j}+A_{j k}\right), \quad \text { for } k \in N(j) \text { and } k \notin N(i)
$$

and

$$
A_{l j}^{b}=-\frac{1}{2} \omega_{l j} s_{j i l}+h^{2}\left(A_{i j}+A_{l i}\right), \quad \text { for } l \in N(i) \text { and } l \notin N(j),
$$

where $\omega_{i_{1} i_{2}}$ is the vorticity in the DEC sense, computed at the common node of cells $i_{1}$ and $i_{2}$, if $i_{1}$ and $i_{2}$ have a common node and $\omega_{i_{1} i_{2}}=0$ if they don't; also $s_{i_{1} i_{2} i_{3}}=1$ if the triplet of cells $i_{1}, i_{2}, i_{3}$ is oriented counter-clockwise and $s_{i_{1} i_{2} i_{3}}=-1$ otherwise. 
Now the equations for the commutator $\left[A^{b}, A\right]$ become

$$
\left[A^{b}, A\right]_{i j}=-\frac{1}{2} \sum_{k \in N(j)} A_{k j} \omega_{i k} s_{i k j}+\frac{1}{2} \sum_{l \in N(i)} A_{i l} \omega_{l j} s_{j i l}-h^{2} \sum_{k \in N(j)} A_{j k}^{2}+h^{2} \sum_{l \in N(i)} A_{i l}^{2} .
$$

If $k \in N(l)$ then $\omega_{i k}=\omega_{l j}$, so only two $\omega$ 's are present in the expression above. Let's denote them by $\omega_{-}$and $\omega_{+}$(see Fig. 2.7) and write

$$
\left[A^{b}, A\right]_{i j}=-\omega_{-} \frac{A_{j k_{1}}+A_{i l_{1}}}{2}-\omega_{+} \frac{A_{k_{2} j}+A_{l_{2} i}}{2}+Q_{i}-Q_{j}
$$

where $Q_{i}=h^{2} \sum_{l \in N(i)} A_{i l}^{2}$.

As we know, $\omega_{-} / h^{2}$ and $\omega_{+} / h^{2}$ approximate the values $\omega\left(x_{-}\right), \omega\left(x_{+}\right)$of vorticity at the corresponding nodes. Also, $A_{i j} \approx v_{i j} / h$. Now suppose the pair of cells $C_{i}$ and $C_{j}$ is oriented along the $y$ direction (see Fig. 2.7 again) and $v=\left(v_{1}, v_{2}\right)$. Let's denote $A_{i j} \approx-v_{2} / h$ and

$$
\begin{aligned}
& A_{i k_{1}} / h=v_{1}^{--} A_{k_{2} i} / h=v_{1}^{+-} \\
& A_{j k_{1}} / h=v_{1}^{-+} A_{k_{2} j} / h=v_{1}^{++}
\end{aligned}
$$

Now, the discrete discrete Euler equation implies

$$
\dot{v}_{2}+\frac{1}{4}\left(\omega\left(x_{-}\right)\left(v_{1}^{--}+v_{1}^{-+}\right)+\omega\left(x_{+}\right)\left(v_{1}^{+-}+v_{1}^{++}\right)\right)+P_{i}-P_{j}=0
$$

where $P$ is some discrete function, playing the role of pressure. This equation, together with the equations for every pair $i$ and $j$, is a discrete version of the twodimensional Euler equations written in the form

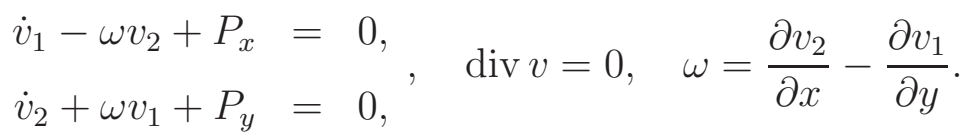

The discretization we have obtained on the regular grid coincides with the HawlowWelsh scheme (see [17]) and Equ. (2.63) is a Crank-Nicolson time update. So our variational scheme is an extension of this approach to arbitrary grids, offering the added bonus of providing a geometric picture to these numerical update rules. Figure 2.8 
57

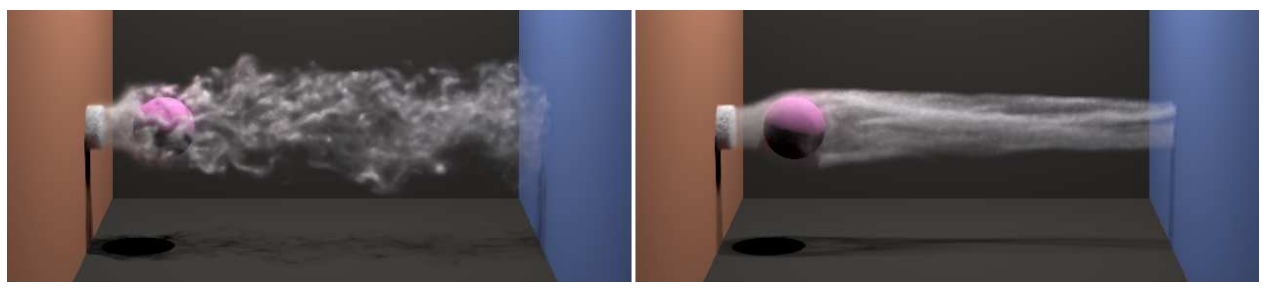

Figure 2.8: A sample 3-D fluid simulation

shows a result of a simulation of a three-dimensional fluid, see [30] for details. 


\section{Chapter 3}

\section{Conclusion}

To our knowledge, the geometric discretization of diffeomorphisms and their geodesics described in this dissertation represents the first attempt at establishing fully Eulerian forms of variational integrators for dynamical systems (for other ideas in the same direction, see, e.g., [12]). As such, our discrete geometric approach is likely to be applicable to a number of other contexts. In this last chapter, we discuss some possible extensions of our doctoral work. Our idea of tracking the motion of mass moving around instead of tracking individual particles shares similarities with recent work, for instance, in the study of invariant manifolds [13], or with the notion of generalized flows introduced by Brenier [9]. Regarding the specific case of fluids, the general philosophy of tracking the transfers of mass has been also used (by Brenier [9], and DiPerna and Majda [16] for instance) to construct extended notions of solutions to the Euler equations, as the problem of global existence of weak solutions to the Euler equations remains open. We describe what we believe are the most challenging avenues to explore in the future.

\subsection{Shortest Paths and Generalized Flows}

Since the motion of an ideal incompressible fluid is described by a geodesic on the group SDiff of volume-preserving diffeomorphisms, the geometric properties of SDiff (along with the resulting properties of Euler equation's solutions) have been studied at length. A natural question raised by our work is whether our finite-dimensional group 
$\mathcal{D}(\mathbb{M})$, used as a discrete substitute for SDiff, preserves these geometric structures. In this section, we will restrict our discussions to metric properties of SDiff related to the Shortest Path Problem (SPP) which amounts to minimize the path length connecting two diffeomorphisms.

\subsubsection{Shnirelman's example}

As showed by Ebin and Marsden in [18], the Shortest Path Problem always has a unique solution locally, i.e., for any pair of diffeomorphisms $g$ and $h$ which are sufficiently close in an appropriate Sobolev norm, there is a unique shortest path connecting $g$ and $h$. However, the Shortest Path Problem may not have a solution in case $n=3$. Next we briefly describe an example, constructed by A. Shnirelman in [35], of a diffeomorphism of the unit three-dimensional cube, which cannot be connected with the identity by a path of shortest length.

Let $I^{n}=[0,1]^{n}$ be an $n$-dimensional unit cube. To find a diffeomorphism for which the SPP is not solvable we will use the fact that the geometry of $\operatorname{SDiff}\left(I^{n}\right)$ is drastically different in cases $n=2$ and $n=3$. Let's consider a diffeomorphism $h \in \operatorname{SDiff}\left(I^{3}\right)$, which has the form

$$
h\left(x_{1}, x_{2}, x_{3}\right)=\left(H\left(x_{1}, x_{2}\right), x_{3}\right),
$$

where $H \in \operatorname{SDiff}\left(I^{2}\right)$. It was proven by A.I. Shnirelman in [35] that the diameter of $\operatorname{SDiff}\left(I^{n}\right)$ is finite for $n=3$ and infinite for $n=2$. Thus, we can assume that:

$$
\operatorname{dist}_{\mathrm{SDiff}\left(I^{3}\right)}(\mathrm{Id}, h)<\operatorname{dist}_{\mathrm{SDiff}\left(I^{2}\right)}(\mathrm{Id}, H) .
$$

We will call the set of diffeomorphisms from $\operatorname{SDiff}\left(I^{n}\right)$ satisfying properties (3.1) and (3.2) the "Shnirelman's class".

It turns out that for any diffeomorphism of the Shnirelman's class, the global Shortest Path Problem is not solvable. Indeed, for any path $\left\{g_{t}\right\}$ connecting $h$ to the 
identity we can construct a (discontinuous) flow $\tilde{g}_{t}$ by rescaling $g_{t}$ in direction $x_{3}$ :

$$
\text { when } 0<x_{3}<\frac{1}{2} \quad \begin{aligned}
& \tilde{g}_{t}^{i}\left(x_{1}, x_{2}, x_{3}\right)=g_{t}^{i}\left(x_{1}, x_{2}, 2 x_{3}\right), \quad \text { for } i=1,2 \\
& \tilde{g}_{t}^{3}\left(x_{1}, x_{2}, x_{3}\right)=\frac{1}{2} g_{t}^{3}\left(x_{1}, x_{2}, 2 x_{3}\right), \\
& \text { when } \frac{1}{2}<x_{3}<1
\end{aligned} \quad \begin{aligned}
& \tilde{g}_{t}^{i}\left(x_{1}, x_{2}, x_{3}\right)=g_{t}^{i}\left(x_{1}, x_{2}, 2-2 x_{3}\right), \quad \text { for } i=1,2 \\
& \tilde{g}_{t}^{3}\left(x_{1}, x_{2}, x_{3}\right)=\frac{1}{2} g_{t}^{3}\left(x_{1}, x_{2}, 2-2 x_{3}\right) .
\end{aligned}
$$

Here $g_{t}^{i}(x)$ is the $i$-th component of $g_{t}(x)$. This rescaled flow also connects $h$ to the identity, however, the vertical component of its action is less then the vertical component of the action along $g_{t}$. Condition (3.2) guarantees that the vertical component of the action is not zero, therefore, $\mathcal{L}\left\{\tilde{g}_{t}\right\}<\mathcal{L}\left\{g_{t}\right\}$. And since for any extremal trajectory $g_{t}$ we have $\mathcal{L}\left\{g_{t}\right\}_{0}^{1}=\frac{1}{2}\left(\ell\left\{g_{t}\right\}_{0}^{1}\right)^{2}$, the length $\ell\left\{g_{t}\right\}$ cannot be minimal.

\subsubsection{Generalized flows}

Since the Shortest Path Problem doesn't always have a global solution in $\operatorname{SDiff}\left(I^{3}\right)$, we can wonder whether there exists a wider class of flows for which the SPP is always solvable. A natural concept of generalized solutions to the SPP was found by Y. Brenier (see [8]). He defined a "generalized flow" as a probability measure on the set of all paths, therefore allowing particles to conceptually split and collide in complicated ways.

Definition 12. A generalized flow on $M$, connecting a diffeomorphism $g \in \operatorname{SDiff}(M)$ with the identity, is a probability measure $\mu$ on the set of continuous paths in $M$ satisfying the following conditions:

1. Volume preservation: for every $A \subset M$ and $t_{0} \in[0,1]$ :

$$
\mu\left\{x(\cdot) \mid x\left(t_{0}\right) \in A\right\}=\operatorname{vol}(A) .
$$

2. Finiteness of action: the action of $\mu$ is the average action along the paths:

$$
\mathcal{L}\{\mu\}=\int\left(\int_{0}^{1}\|\dot{x}(t)\|^{2} d t\right) d \mu<\infty .
$$


3. Boundary condition: for $\mu$-almost all paths, $x(1)=g(x(0))$.

Any regular flow $g_{t}$ can be considered as a generalized flow. In this case the measure $\mu$ is concentrated on the trajectories of $g_{t}$, and it is defined as

$$
\mu(X)=\operatorname{vol}\left\{x \in M \mid g_{t}(x) \in X\right\}
$$

As proved by Y. Brenier, the SPP is always solvable in this class of generalized flows, i.e., for any $g \in \operatorname{SDiff}(M)$ there is a generalized flow $\mu$ realizing the minimum of action. It is also shown in [35] that any generalized flow can be approximated together with its action by regular flows. This means, in particular, that the distance

from a diffeomorphism $g \in \operatorname{SDiff}(M)$ to the identity is equal to $(2 \mathcal{L}\{\mu\})^{\frac{1}{2}}$, where $\mu$ is a minimal generalized flow, connecting $g$ with the identity. This fact was used by A. I. Shnirelman (see [34]) to estimate the diameter of $\operatorname{SDiff}\left(I^{3}\right)$.

In many aspects, the minimal generalized flows are similar to the solutions of the Euler equation. For example, there exists a function $p$ such that for almost every particle its acceleration is equal to the gradient $\nabla p$, so $p$ can be considered as a generalized pressure. Also, certain classes of generalized flows were used by Shnirelman to construct weak solutions to the Euler equation exhibiting interesting properties, such as monotone energy decay etc.

\subsubsection{Discrete generalized flows}

Our discrete model of fluid flow is obtained though a variational problem. However, we cannot formulate a variational problem, since we only consider paths satisfying the non-holonomic constraints, but their variations don't need to satisfy the NHC. Thus, we don't have a space where we can formulate a discrete analog of the Shortest Path Problem. The non-holonomic constraints appear in our work as a way to construct a discrete version of the inner product. Indeed, any matrix $A_{h}$ can be considered as an approximation to a vector field $v$ as long as $A_{h} \varphi_{h} \rightarrow-L_{v} \varphi$ when $\varphi_{h} \rightarrow \varphi$, but a-priori we don't know how to construct an approximation to $\int v^{2}$ using the elements of $A$. So we restrict the matrix $A$ to belong to the constrained set $\mathcal{S}$, where we know 
how to treat the elements $A_{i j}$ as (quasi) fluxes and therefore how to compute $\int v^{2}$. However, in order to get a discrete version of the Shortest Path Problem, we have to relax the non-holonomic constraints and construct a discrete inner product for arbitrary matrices.

If we can relax the non-holonomic constraints and consider the Shortest Path Problem, the next question one can ask is: what happens when the end-point converges to a diffeomorphism from the Shnirelman's class? We can expect the minimum to always exist in the finite dimensional case and, therefore, our discrete flow to be an approximation of a Brenier's generalized flow.

Our construction exhibits many similarities with the concept of a generalized flow. Indeed, it is based on the idea of tracking a function (or a mass) instead of trajectories of individual particles. For example, if we use (as mentioned in the remark on Section 2.1.1) a doubly-stochastic matrix $q(t)$ to discretize a diffeomorphism $g_{t}$ at time $t$, then for any cell $C_{j}$ we know the probability $q_{i j}$ for a point $x \in C_{j}$ to appear in cell $C_{i}$ at time $t$. Thus a one-parameter family of matrices $q_{t}$ can be considered as a discretization of a generalized flow as well. The question of how to correctly approximate the action of a generalized flow remains, however, open.

\subsection{Symplecticity}

Variational integrators in the Lagrangian setting are known to be symplectic, in that they conserve the canonical symplectic structure of the dynamical system they discretize. An interesting continuation of our work would therefore be to prove (or disprove) that our resulting discrete Euler-Lagrange equations for an inviscid Euler fluid preserves a discrete symplectic form in time. Numerical tests, showing good long-time behavior of the energy, seem to indicate symplecticity of our scheme. 


\subsection{Optimal Transport}

Recently there has been a huge amount of interest in optimal transport theory, in particular due to work of Brenier. These advances partially stem from the fact transport theory is applicable to vast array of practical problems that include registration of medical datasets as studied by Tannenbaum et al. [21]. As the configuration space used in our work can be seen as discrete maps on the space of measures, our method should provide an interesting discretization of optimal transport theory, leading not only to further geometric insights on transport theory but also to efficient computational schemes.

\subsection{Other Future Work}

\subsubsection{Magnetohydrodynamics}

We also wish to use our approach to geometrically derive discrete equations of motion for magnetohydrodynamics (MHD). An existing form-based discretization of E\&M [36], fully compatible with our setup, should be easy to incorporate in our framework.

\subsubsection{Viscosity and subscale modeling}

The discrete Euler-Lagrange equations we derived have also remarkable similarities with subscale modeling techniques such as the $\alpha$-model, where two codependent velocity fields are simultaneously advected to preserve the $H^{1}$ norm of the velocity. We

believe that the modeling of fine scales can be included in our approach to better capture the energy cascading and inertial range of viscous fluids. 


\section{Bibliography}

[1] Arnold, V. I., [1966], Sur la géométrie différentielle des groupes de Lie de dimenson infinie et ses applications à l'hydrodynamique des fluides parfaits, Ann. Inst. Fourier, Grenoble, 16:319-361.

[2] Arnold, V. I., [1969], Hamiltonian character of the Euler equations of the dymnamics of solids and of an ideal fluid, Uspekhi Mat. Nauk, 24:225-226.

[3] Arnold, D.N., R. S. Falk, and R. Winther [2006], Finite element exterior calculus, homological techniques, and applications, Acta Numerica, 15, 1-155.

[4] Arnold, V. I. and B. Khesin [1992], Topological methods in hydrodynamics, Ann. Rev. Fluid Mech., 24, 145-166.

[5] Arnold, V. I. and B. Khesin [1998], Topological methods in hydrodynamics, Springer-Verlag.

[6] Bochev, P. B. and J. M. Hyman [2005], Principles of mimetic discretizations of differential operators, Preprint LA-UR-05- 4244.

[7] Bossavit, A. [1998], Computational Electromagnetism. Academic Press (Boston).

[8] Brenier, Y. [1989], The least action principle and the related concept of generalized flows for incompressible perfect fluids, J. Amer. Math. Soc. 2, no. 2, $225-255$.

[9] Brenier, Y. [1999], Minimal geodesics on groups of volume-preserving maps and generalized solutions of the Euler equations, Comm. Pure Appl. Math. 52, 411452. 
[10] Bretherton, F. P. [1970], A note on Hamilton's principle for perfect fluids, J. Fluid Mech. 44, 99-31.

[11] Cendra, H. and J. E. Marsden [1987], Lin constraints, Clebsch potentials and variational principles, Physica D 27, 63-89.

[12] Cotter, C. J. and D. D. Holm [2009], Continuous and Discrete Clebsch Variational Principles, Foundations of Computational Mathematics 9(2), 221-242.

[13] Dellnitz, M., A. Hohmann, O. Junge, and M.Rumpf [1997], Exploring invariant sets and invariant measures, CHAOS: An Interdisciplinary Journal of Nonlinear Science 7(2), 221-228.

[14] Desbrun, M., E. Kanso, and Y. Tong, [2005], Discrete differential forms for computational modeling. Chapter in ACM SIGGRAPH Course Notes on Discrete Differential Geometry.

[15] Desbrun, M., A. N. Hirani, and J. E. Marsden [2003], Discrete exterior calculus for variational problems in computer vision and graphics, Proc. CDC 42, 533538.

[16] DiPerna, R. G. and A. G. Majda [1987], Oscillations and concentrations in weak solutions of the incompressible fluid equations, Math. Physics 108, 667-689.

[17] Harlow, F. H. and J. E. Welch [1965], Numerical calculation of time-dependent viscous incompressible flow of fluid with free surface, Physics of Fluids 8(12), 2182-2189.

[18] Ebin, D. G. and J. E. Marsden [1970], Groups of diffeomorphisms and the motion of an incompressible fluid, Ann. of Math., 92, 102-163.

[19] Elcott, S., Y. Tong, E. Kanso, P. Schröder, and M. Desbrun [2007], Stable, circulation-preserving simplicial fluids, ACM Transactions on Graphics, 26(1), Art. 4. 
[20] Hairer, E., C. Lubich, and G. Wanner [2006], Geometric Numerical Integration: Structure-Preserving Algorithms for Ordinary Differential Equations. SpringerVerlag.

[21] Haker, S., L. Zhu, A. Tannenbaum, and S. Angenent [2004], Optimal Mass Transport for Registration and Warping, International Journal on Computer Vision $60(3), 225-240$.

[22] Hou, T. Y. and Z. Lei [2009], On the Stabilizing Effect of Convection in 3D Incompressible Flow, Commun. Pure Appl. Math., 62(4), 501-564.

[23] Hirani, A. [2003], Discrete Exterior Calculus, PhD thesis, California Institute of Technology.

[24] Koopman, B. O. [1931], Hamiltonian Systems and Transformations in Hilbert Spaces, Proc. Nat. Acad. Sci. (USA), 17, 315-318.

[25] Marsden, J. E. and T. S. Ratiu [1999], Introduction to Mechanics and Symmetry, 17 of Texts in Applied Mathematics, vol. 17; 1994, Second Edition, 1999. Springer-Verlag.

[26] Marsden, J. E., T. S. Ratiu, and S. Shkoller [2000], The geometry and analysis of the averaged Euler equations and a new diffeomorphism group, Geom. Funct. Anal., 10(3), 582-599.

[27] Marsden, J. E. and J. Scheurle [1993], The reduced Euler-Lagrange equations, Fields Institute Comm., 1, 139-164.

[28] Marsden, J. E. and A. Weinstein [1983], Coadjoint orbits, vortices and Clebsch variables for incompressible fluids, Physica D 7, 305-323.

[29] Marsden, J. E. and M. West [2001], Discrete mechanics and variational integrators, Acta Numerica, 10, 357-515.

[30] Mullen, P., K. Crane, D. Pavlov, Y. Tong, M. Desbrun [2009], Energy-preserving integrators for fluid simulation, ACM Trans. on Graphics 2009 
[31] Munkres, J. R. [1984], Elements of Algebraic Topology, Addison-Wesley, Menlo Park, CA.

[32] Newcomb, W. A. [1962], Lagrangian and Hamiltonian methods in Magnetohydrodynamics, Nuc. Fusion Suppl. part 2, 451-463.

[33] Sen, S., S. Sen, J. C. Sexton, and D. H. Adams [2000], Geometric discretization scheme applied to the abelian Chern-Simons theory, Phys. Rev. E 61(3), 31743185.

[34] Shnirelman, A. I. [1994], Generalized fluid flows, their approximation and applications, Geom. and Funct. Analysis, 4, no. 5, 586-620.

[35] Shnirelman, A. I. [1997], On the non-uniqueness of weak solution of the Euler equations, Comm. Pure Appl. Math. 50(12), 1261-1286.

[36] Stern, A., Y. Tong, M. Desbrun, and J. E. Marsden [2008], Variational Integrators for Maxwells Equations with Sources, Progress in Electromagnetics Research Symposium (PIERS) 4(7), 711-715. 